\title{
Sur le théorème de division de Weierstrass
}

par

\author{
J ACQUES CHAUMAT (Orsay) \\ et ANNE-MARIE CHOLLET (Lille)
}

Abstract. We prove a Weierstrass division formula for $C^{\infty}$ Whitney jets $\bar{\partial}$-flat on arbitrary compact subsets of the complex plane. We also give results for Carleman classes.

Introduction. Une des motivations de ce travail est le théorème classique de Weierstrass dont il existe plusieurs formes équivalentes. Celle qui sera évoquée ici s'exprime en termes du polynôme générique de degré $m$ de la variable complexe $z$,

$$
P_{m}(z, \lambda)=z^{m}+\lambda_{m} z^{m-1}+\ldots+\lambda_{2} z+\lambda_{1} .
$$

Pour toute fonction $F$ holomorphe au voisinage de 0 dans $\mathbb{C}^{1+s}$, on a

$$
F(z, t)=P_{m}(z, \lambda) Q(z, \lambda, t)+\sum_{k=0}^{m-1} z^{k} R_{k}(\lambda, t),
$$

où $Q$ est une fonction holomorphe au voisinage de 0 dans $\mathbb{C} \times \mathbb{C}^{m} \times \mathbb{C}^{s}$ et $R_{k}$, $0 \leq k \leq m-1$, des fonctions holomorphes au voisinage de 0 dans $\mathbb{C}^{m} \times \mathbb{C}^{s}$.

$\mathrm{Si}$, maintenant, $\Omega$ est un ouvert de $\mathbb{R}^{s}$ et $f$ une fonction de classe $C^{\infty}$ sur $\mathbb{R} \times \Omega$ on a

$$
f(x, t)=P_{m}(x, \lambda) Q(x, \lambda, t)+\sum_{k=0}^{m-1} x^{k} R_{k}(\lambda, t),
$$

où $Q$ est de classe $C^{\infty}$ sur $\mathbb{R} \times \mathbb{C}^{m} \times \Omega$ et, pour tout $k, 0 \leq k \leq m-1, R_{k}$ esi de classe $C^{\infty}$ sur $\mathbb{C}^{m} \times \Omega$. Il s'agit là d'un théorème également classique de B. Malgrange [11] dont différentes preuves ont été données, en particulier, par G. Glaeser, S. Lojasiewicz, J. Mather et L. Nirenberg. On trouvera des références dans [14] et [15].

La preuve donnée par J. Mather a permis à M. D. Bronshteĭn [3] d'étendre la formule de division aux classes de Gevrey. Dans une autre direction,

1991 Mathematics Subject Classification: 26E10, 30E20,32B05, 46E35, 46J15.

Key words and phruses: division, Weierstrass, Whitney jets, non-quasi-analytic. 
S. Bell et D. Catlin [2] ont modifé une preuve de L. Nirenberg [15] et donné une version de ce théorème adaptée aux fonctions holomorphes dans un demi-plan qui se prolongent de façon $C^{\infty}$ jusqu'au bord. Indépendamment, une idée voisine a été exploitée par K. Diederich et J. Fornæess [8].

Les auteurs développent ici une méthode permettant d'écrire une formule de division valable sur un compact arbitraire $K$ du plan complexe et pour des classes de jets de Whitney $\bar{\partial}$-plats sur $K$. Ils se situent ainsi dans un cadre unifiant les travaux précédents. L'introduction des classes de jets de Whitney dans cette théorie n'est pas artificielle, bien au contraire. On sait en effet que, si un compact $K$ est l'adhérence d'un ouvert à bord Lip1, les jets de Whitney $C^{\infty}$ et $\bar{\partial}$-plats sur $K$ s'identifient à $A^{\infty}(K)$, la classe des fonctions holomorphes à l'intérieur de $K$ qui se prolongent continûment ainsi que leurs dérivées au bord de $K$, et que si $K$ est un intervalle $[a, b]$ de $\mathbb{R}$, les jets de Whitney $C^{\infty}$ et $\bar{\partial}$-plats sur $K$ s'identifient aux fonctions de classe $C^{\infty}$ au sens usuel sur $[a, b]$. Ainsi, dans ce travail, tout théorème de division établi au sens des jets aura deux corollaires que l'on pourra écrire dans chacune de ces situations. Par souci de brièveté, on ne donnera pas le détail des preuves dans chaque cas.

Dans une première partie, si $K$ est un compact arbitraire de $\mathbb{C}$, on établit, en utilisant la formule de Cauchy et une fonction troncature inspirée de J. Mather et de M. D. Bronshteĭn, une formule de division pour une fonction $g$ de classe $C^{\infty}$ dans $\mathbb{C}$ et à support compact au voisinage de $K$. C'est la proposition 4 . La fonction $Q$ et les fonctions $R_{k}, 0 \leq k \leq m-1$, sont données par des intégrales portant sur $\bar{\partial} g$ dont les estimations se font simplement. On a alors un théorème de division (théorème 7) sans paramètres au sens des jets sur un compact arbitraire $K$ de $\mathbb{C}$. En application, on mentionne les résultats concernant un compact $K$ à bord Lip1 ou un intervalle compact de $\mathbb{R}$.

On sait bien que, pour obtenir un théorème de division à paramètres, il importe d'avoir un quotient $Q$ et des restes $R_{k}, 0 \leq k \leq m-1$, dépendant linéairement et continûment de la fonction dividende donnée. Si $K$ est un compact arbitraire de $\mathbb{C}$, il est donc essentiel d'avoir un procédé d'extension linéaire des jets de Whitney $C^{\infty}$ sur $K$ en fonction $C^{\infty}$ à support compact dans $\mathbb{C}$. Ceci n'est pas toujours possible et nous introduisons des espaces de Banach de jets $\left\{p ! M_{p} ; C\right\}_{K}^{+}$pour lesquels il existe toujours un opérateur linéaire continu d'extension dans $C^{\infty}(\mathbb{C})$. On a alors le théorème de division 13 des jets de Whitney $C^{\infty}(K)$ et $\bar{\partial}$-plats sur $K$ avec paramètres dont on déduit le théorème de $\mathrm{B}$. Malgrange avec paramètres pour les fonctions $C^{\infty}$ sur un intervalle compact de $\mathbb{R}$ (théorème 14) et un théorème de division pour les fonctions de $A^{\infty}(K)$ dans le cas d'un compact à bord Lipl (théorème 15). On retrouve, si $K$ est le demi-disque unité de $\mathbb{C}$, le résultat de $\mathrm{S}$. Bell et $\mathrm{D}$. Catlin mentionné plus haut.
La deuxième partie concerne les classes de jets de Whitney fortement non quasi-analytiques. Le théorème 20 est un théorème de division à paramètres dans ces classes. Comme précédemment, on a deux corollaires dans le cas d'un compact à bord Lip1 et dans le cas d'un intervalle compact de $\mathbb{R}$. On retrouve alors, comme cas particulier, le résultat précité de $\mathrm{M}$. D. Bronshteŭn sur les classes de Gevrey. Ce théorème fait apparaître une perte de régularité par rapport aux paramètres.

Un dernier paragraphe est consacré à des applications du théorème 20; on donne cles résultats du type "théorème de Newton". Ceux-ci permettent d'établir que la perte de régularité par rapport aux paramètres du théorème 20 est optimale.

\section{Régularité $C^{\infty}$}

1. DÉFinitions ET NOTATIONS. Soit $\left\{M_{p}\right\}_{p \geq 0}$ une suite de réels positifs vérifiant les propriétés suivantes :

(1.1) $\quad M_{0}=1$ et $\left\{M_{p}\right\}_{p \geq 0}$ est logarithmiquement convexe,

$$
\lim _{p \rightarrow \infty} M_{p}^{1 / p}=\infty
$$

On considère maintenant la condition

$$
\sum_{p=1}^{\infty} \frac{M_{p-1}}{p M_{p}}<\infty .
$$

On sait que (1.1) et (1.3) entrainent (1.2) et que, pour tout $j$ et tout $k$, on a

$$
M_{j} M_{k} \leq M_{j+k} \text {. }
$$

Soit $J=\left(j_{1}, \ldots, j_{s}\right)$, un multi-indice dans $\mathbb{N}^{s}$. On note $|J|=j=j_{1}+$ $\ldots+j_{s}$ et $J !=j_{1} ! \ldots j_{s} !$. Pour tout $x=\left(x_{1}, \ldots, x_{s}\right)$ de $\mathbb{R}^{s}$, on pose $x^{J}=$ $x_{1}^{j_{1}} \ldots x_{s}^{j_{n}}$ et on note $|x|$ la norme euclidienne de $x$. Pour tout compact $K$ de $\mathbb{R}^{s}$ et tout $x$, on note $d(x, K)$ la distance de $x$ à $K$ et $V(K)=\left\{x \in \mathbb{R}^{s}\right.$ : $d(x, K)<1\}$.

Soit $K$ un sous-ensemble compact de $\mathbb{R}^{s}$. Un jet $F$ sur $K$ est la donnée d'une suite $\left\{F^{J}(\zeta) ; \zeta \in K, J \in \mathbb{N}^{s}\right\}$ de fonctions continues sur $K$ à valeurs dans $\mathbb{C}$.

Pour un multi-indice $L$ de longueur $l$ de $\mathbb{N}^{s}$, on définit la $L$-ième dérivée du jet $F$ par

$$
D^{L} F=\frac{\partial^{l} F}{\partial x_{1}^{l_{1}} \cdots \partial x_{s}^{l_{h}}}=\left\{F^{J+L}(\zeta): \zeta \in K, J \in \mathbb{N}^{s}\right\}
$$

Pour un opérateur différentiel $P$ à coefficients continus, on définit de manière analogue le jet $P F$. On dira que $F$ est $P_{\text {-plat }}$ si le jet $P F$ est identiquement nul. 
Pour tout jet $F$, tout $\zeta$ de $K$ et tout entier $p$, on définit le polynôme de Taylor de $F$, pour tout $x$ de $\mathbb{R}^{8}$, par

$$
T_{\zeta}^{p} F(x)=\sum_{J,|J| \leq p} \frac{1}{J !}(x-\zeta)^{J} F^{J}(\zeta)
$$

et, pour tout entier $p$, pour tout multi-indice $J$ avec $j \leq p$ et tout $(\zeta, x)$ de $K \times K$

$$
\text { (1.6) } \quad R_{\zeta}^{J, p} F(x)=F^{J}(x)-\sum_{L,|J+L| \leq p} \frac{1}{L !}(x-\zeta)^{L} F^{J+L}(\zeta) \text {. }
$$

On rappelle qu'un jet $F$ est un jet de Whitney sur. $K$ de classe $C^{\infty}$, et on note $F \in C^{\infty}(K)$, si on a, pour tout entier $p$ et pour tout multi-indice $J$ avec $j \leq p$

$$
R_{\zeta}^{J, p} F(x)=o\left(|\zeta-x|^{p-j}\right)
$$

lorsque $|\zeta-x|$ tend vers 0 avec $(\zeta, x)$ dans $K \times K$.

Si $f$ désigne une fonction de classe $C^{\infty}$ dans $\mathbb{R}^{s}$, on note, pour tout multi-indice $L$ de longueur $l$ et tout $x$ de $\mathbb{R}^{s}$,

$$
D^{L} f(x)=\frac{\partial^{l} f}{\partial x_{1}^{l_{1}} \ldots \partial x_{s}^{l_{a}}}(x) .
$$

Bien évidemment, $f$ définit naturellement un jet de Whitney de classe $C^{\infty}$ sur $K$ que l'on note encore $f$.

On sait, d'après le théorème d'extension de Whitney [11], [14] que, pour tout jet $F$ de $C^{\infty}(K)$, il existe une fonction $f$ de classe $C^{\infty}$ dans $\mathbb{R}^{s}$, à support compact, telle que, pour tout multi-indice $L$ de $\mathbb{N}^{s}$ et tout $\zeta$ de $K$, on ait

On écrit encore

$$
F^{L}(\zeta)=D^{L} f(\zeta)
$$

$$
f=F, \quad \text { au sens des jets sur } K \text {. }
$$

2. LEMME [10]. Soit $\left\{M_{p}\right\}_{p \geq 0}$ une suite vérifiant (1.1) et (1.3). Soit $\Omega$ un sous-ensemble non vide de $\mathbb{R}^{s}$ et a un réel strictement positif. Il existe une fonction $\chi_{\Omega}$ de classe $C^{\infty}$ dans $\mathbb{R}^{s}$ à valeurs positives ou nulles et vérifiant les propriétés suivantes:

$$
\begin{array}{ll}
\chi_{\Omega}(x)=1 & \text { si } d(x, \Omega) \leq \frac{1}{2} a, \\
\chi_{\Omega}(x)=0 & \text { si } d(x, \Omega) \geq a .
\end{array}
$$

Pour tout multi-indice $L$ de $\mathbb{N}^{s}$ de longueur $l$ et tout $x$ de $\mathbb{R}^{s}$, on a

$$
\left|D^{L} \chi_{\Omega}(x)\right| \leq A_{0}^{l} l ! M_{l} a^{-l}
$$

Ici $A_{0}$ est une constante qui ne dépend que de la dimension s et de la suite $\left\{M_{p}\right\}_{p \geq 0}$.
On note, pour tout réel $A_{1}>0$,

$$
O\left(A_{1}\right)=\left\{(z, \zeta) \in \mathbb{C} \times V(K) \backslash K: d(z, K)<A_{1} d(\zeta, K)\right\}
$$

Soit $m$ un entier strictement positif. On note $P_{m}$ le polynôme défini, pour tout $\lambda=\left(\lambda_{1}, \ldots, \lambda_{m}\right)$ de $\mathbb{C}^{m}$ et tout $z$ de $\mathbb{C}$, par

$$
P_{m}(z, \lambda)=z^{m}+\lambda_{m} z^{m-1}+\ldots+\lambda_{2} z+\lambda_{1} \text {. }
$$

Le lemme suivant est une adaptation d'un lemme de J. Mather [15] et M. D. Bronshteln [3].

3. LEMME. Soit $K$ un compact de $\mathbb{C},\left\{M_{p}\right\}_{p \geq 0}$ une suite de réels vérifiant les propriétes (1.1) et (1.3). Soit $m$ un entier strictement positif. Il existe des constantes $A_{2}$ et $A_{3}$ ne dépendant que de la suite $\left\{M_{p}\right\}_{p} \geq 0$, de l'entier $m$ et du compact $K$ et il existe une fonction $\varphi$ de classe $C^{\infty}$ sur $O(1) \times \mathbb{C}^{m}$ telles que les propriétés suivantes soient vérifiées:

$$
\begin{array}{ll}
\varphi(z, \zeta, \lambda)=1 & \text { si } d(z, K)<\frac{1}{64} d(\zeta, K), \\
\varphi(z, \zeta, \lambda)=0 & \text { si } d(z, K)>\frac{3}{4} d(\zeta, K) .
\end{array}
$$

Pour tout multi-indice $L \in \mathbb{N}^{2 m}$ de longueur $l$ et tout $(z, \zeta, \lambda)$ de $O(1) \times \mathbb{C}^{m}$, on a

$$
\left|D_{\lambda}^{L} \frac{\partial}{\partial \bar{z}} \varphi(z, \zeta, \lambda)\right| \leq A_{2}^{l} l ! M_{l} d(\zeta, K)^{-(m l+1)}
$$

En tout point $(z, \zeta, \lambda)$ de $O(1) \times \mathbb{C}^{m}$ où $\frac{\partial}{\partial z} \varphi(z, \zeta, \lambda)$ est different de 0 , on a

$$
\left|P_{m}(z, \lambda)\right| \geq A_{3} d(\zeta, K)^{m} \text {. }
$$

Preuve. Soit $r \in \mathbb{R}, 0<r<1$ et $j \in \mathbb{N}, 0 \leq j \leq m$. On note

$$
\Gamma_{j, r}=\left\{z \in \mathbb{C}:\left(\frac{1}{4}+\frac{j}{2(m+1)}\right) r \leq d(z, K)<\left(\frac{1}{4}+\frac{j+1}{2(m+1)}\right) r\right\}
$$

(3.5) $\quad N_{j, r}=\left\{\lambda \in \mathbb{C}^{r b}: P_{m}(\cdot, \lambda)\right.$ ne s'annule pas dans $\left.\Gamma_{j, r}\right\}$.

On a, pour tout $r$,

$$
\bigcup_{j=m}^{m} N_{j, r}=\mathbb{C}^{m}
$$

En effet, s'il existait $\lambda \in \mathbb{C}^{m} \backslash \bigcup_{j=0}^{m} N_{j, r}$, alors d'après $(3.5), P_{m}(\cdot, \lambda)$ s'annulerait dans chaque $\Gamma_{j, r}, 0 \leq j \leq m$. De là, puisque les $\Gamma_{j, r}$ sont disjoints, le polynôme $P_{m}(\cdot, \lambda)$ aurait $m+1$ racines, ce qui est absurde.

On note maintenant

(3.7) $\quad D_{j, r}^{\prime}=\left\{z \in \mathbb{C}: d(z, K)<\left(\frac{1}{4}+\frac{j+1}{2(m+1)}-\frac{1}{8(m+1)}\right) r\right\}$ 


$$
\text { (3.8) } \begin{aligned}
\Gamma_{j, r}^{\prime}=\left\{z \in \mathbb{C}:\left(\frac{1}{4}+\frac{j}{2(m+1)}+\frac{1}{8(m+1)}\right) r \leq d(z, K)\right. \\
\left.<\left(\frac{1}{4}+\frac{j+1}{2(m+1)}-\frac{1}{8(m+1)}\right) r\right\} .
\end{aligned}
$$

On a donc, pour tout $j$ avec $0 \leq j \leq m$ et tout $r$ avec $0<r<1$,

(3.9) $\quad\left\{z \in \mathbb{C}: d(z, K)<\frac{1}{4} r\right\}$

$$
\subset D_{0, r}^{\prime} \subset D_{j, r}^{\prime} \subset D_{m, r}^{\prime} \subset\left\{z \in \mathbb{C}: d(z, K)<\frac{3}{4} r\right\}
$$

Si on note, pour $\lambda$ dans $\mathbb{C}^{m}, \mu_{k}(\lambda)$ les racines de $P_{m}(r, \lambda)$, on a

$$
P_{m}(z, \lambda)=\prod_{k=1}^{m}\left(z-\mu_{k}(\lambda)\right)
$$

Si $\lambda$ appartient à $N_{j, r}$ alors les racines $\mu_{k}(\lambda)$ n'appartiennent pas à $\Gamma_{j, r}$ Il existe donc une constante $A_{4}$ ne dépendant que de $m$ telle que l'on ait, pour tout $j$ avec $0 \leq j \leq m$, tout $\lambda$ dans $N_{j, r}$ et tout $z$ vérifiant $d\left(z, \Gamma_{j, r}^{\prime}\right)<$ $r /(16(m+1))$,

$$
\left|P_{m}(z, \lambda)\right| \geq A_{4} r^{m}
$$

Puisque l'on a

$$
\left|P_{m}(z, \lambda)-P_{m}\left(z, \lambda^{\prime}\right)\right| \leq\left|\lambda-\lambda^{\prime}\right| \sup _{K} \sum_{k=1}^{m-1}\left|z^{k}\right|
$$

il existe des constantes $A_{5}$ et $A_{6}$ dépendant de $m$ et de $K$ telles que l'on ait, pour tout $j$ avec $0 \leq j \leq m$, tout $\lambda$ vérifiant $d\left(\lambda, N_{j, r}\right)<A_{5} r^{m}$ et tout $z$ vérifiant $d\left(z, \Gamma_{j, r}^{\prime}\right)<r /(16(m+1))$.
(3.11)$$
\left|P_{m}(z, \lambda)\right| \geq A_{6} r^{m} \text {. }
$$

On note, pour tout $r$ avec $0<r<1$,

$$
C_{r}=\{z \in \mathbb{C}: r / 2 \leq d(z, K)<r\} .
$$

On applique maintenant le lemme 2 à

On a

$$
\begin{aligned}
C_{r} & \text { avec } a=\frac{1}{4} r \\
D_{j, r}^{\prime} \quad \text { avec } a & =\frac{1}{16(m+1)} r \\
N_{j, r} \quad \text { avec } a & =A_{5} r^{m} .
\end{aligned}
$$

$$
\bigcup_{k=0}^{\infty} C_{2-k}=V(K) \backslash K
$$

On note, pour tout entier $k \geq 0,\left(\tilde{\chi}_{C_{2-k}}\right)_{k}$ la suite de fonctions définies par

$$
\begin{aligned}
\tilde{\chi}_{C_{1}} & =\chi_{C_{1}} \quad \text { pour } k=0, \\
\tilde{\chi}_{C_{2-k}} & =\left(1-\chi_{C_{1}}\right) \ldots\left(1-\chi_{C_{2-(k-1)}}\right) \chi_{C_{2-k}} \quad \text { pour tout } k>0 .
\end{aligned}
$$

D'après (3.13), on a, pour tout $\zeta \in V(K) \backslash K$,

$$
\sum_{k=0}^{\infty} \tilde{\chi}_{c_{2} \ldots k}(\zeta)=1
$$

D'après les propriétés (2.1) et (2.2) de $\chi_{C_{2-h}},(3.12)$ et (3.14), on a, pour tout $k \geq 0$

$\operatorname{supp} \tilde{\chi}_{C_{2-k}} \subset \operatorname{supp} \chi_{C_{2-k}} \subset C_{2-(k-1)} \cup C_{2-k} \cup C_{2-(k+1)}$.

Soit $\zeta$ un point de $V(K) \backslash K$. Alors il existe un entier $k(\zeta) \geq 0$ tel que l'on ait

(3.15) $\quad 2^{-(k(\zeta)+1)} \leq d(\zeta, K)<2^{-k(\zeta)} \quad$ et donc $\quad \zeta \in C_{2-k(\zeta)}$.

De là, dans le produit définissant $\widetilde{\chi}_{C_{2-k}}$ dans (3.14), il y a, au plus, pour chaque $\zeta$, deux facteurs significatifs, c'est-à-dire différents de 0 ou de 1 .

De même, on définit, pour tout entier $k_{i} \geq 1,\left(\widetilde{\chi}_{N_{j, 2}-k}\right)_{0 \leq j \leq m}$ par

$$
\begin{aligned}
& \tilde{\chi}_{N_{0,2-k}}=\chi_{N_{0,2 \cdots k s}}, \\
& \tilde{\chi}_{N_{j, 2 \cdots k}}=\left(1-\chi_{N_{0,2-k}}\right) \ldots\left(1-\chi_{N_{j-1,2-k}}\right) \chi_{N_{j, 2}-k} \\
& \text { pour tout } j \text { avec } 0 \leq j \leq m .
\end{aligned}
$$

D'après (3.6), on $a$, pour tout $k \geq 0$ et tout $\lambda \in \mathbb{C}^{m}$,

$$
\sum_{j=0}^{m} \widetilde{\chi}_{N_{j, 2-k}}(\lambda)=1 \text {. }
$$

On pose, pour tout $(z, \zeta, \lambda)$ de $O(1) \times \mathbb{C}^{m}$,

$$
\varphi(z, \zeta, \lambda)=\sum_{k=0}^{\infty} \tilde{\chi}_{C_{2-k}}(\zeta) \sum_{j=0}^{m} \chi_{D_{j, 2}^{\prime}(k+3)}(z) \tilde{\chi}_{N_{j, 2-(k+3)}}(\lambda) .
$$

La fonction $\varphi$ est clairement de classe $C^{\infty}$ dans $O(1) \times \mathbb{C}^{m}$.

On a la temarque-clé suivante :

(3.18) dans la sommation en $k$ définissant la fonction $\varphi$, seuls interviennent les indices $k(\zeta)-1, k(\zeta)$ et $k(\zeta)+1$.

On se propose de montrer que la propriété (3.1) est vérifiée.

Si l'on a $d(z, K)<\frac{1}{64} d(\zeta, K)$ allors $d(z, K)<\frac{1}{4} \cdot 2^{-(k(\zeta)+4)}$ et donc, d'après $(3.9), z$ appartient à $D_{j, 2-(m(\zeta)+\infty)}^{\prime}$ pour tout $j$ avec $0 \leq j \leq m$. Puisque l'on a

$$
D_{j, 2-(k(\zeta)+4)}^{\prime} \subset D_{j, 2-(k(\zeta)+3)}^{\prime} \subset D_{j, 2^{-(h(\zeta)+2)}}^{\prime}
$$


on a, pour tout $\lambda$, d'après $(2.1), \varphi(z, \zeta, \lambda)=1$.

Si maintenant on a $d(z, K)>\frac{3}{4} d(\zeta, K)$ alors $d(z, K)>\frac{3}{4} \cdot 2^{-(k(\zeta)+1)}>$ $\frac{3}{4} \cdot 2^{-(k(\zeta)+2)}$ et donc, d'après $(3.7)$ et $(3.9), z$ n'appartient pas à $D_{m, 2^{-(k(\zeta)+2)}}^{\prime}$ Plus précisément, on vérifie aisément que l'on a

$$
d(z, K)>\frac{3}{4} \cdot 2^{-(k(\zeta)+2)}+\frac{1}{16(m+1)} 2^{-(k(\zeta)+2)}
$$

et donc, d'après $(2.2)$ et $(3.13)$, on a, pour tout $\lambda, \varphi(z, \zeta, \lambda)=0$.

On se propose maintenant de contrôler les dérivées de $\varphi$ sur $O(1) \times \mathbb{C}^{m}$. Il existe, d'après (2.3), (3.12), (3.14) et (3.16), une constante $A_{7}>1$ ne dépendant que de la suite $\left\{M_{p}\right\}_{p \geq 0}$ et de l'entier $m$ telle que l'on ait :

- pour tout multi-indice $\alpha$ de $\mathbb{N}^{2}$ de longueur $|\alpha|$ et tout $\zeta$ de $\mathbb{C}$,

$$
\left|D^{\alpha} \widetilde{\chi}_{C_{2-k}}(\zeta)\right| \leq A_{7}^{|\alpha|}|\alpha| ! M_{|\alpha|}\left(\frac{1}{2^{k+2}}\right)^{|\alpha \alpha|}
$$

- pour tout multi-indice $\beta$ de $\mathbb{N}^{2}$ de longueur $|\beta|$ et tout $z$ de $\mathbb{C}$,

$$
\left|D^{\beta} \widetilde{\chi}_{D_{j, 2-k}^{\prime}}(z)\right| \leq A_{7}^{|\beta|}|\beta| ! M_{|\beta|}\left(\frac{1}{16(m+1) 2^{k}}\right)^{|\beta|}
$$

- pour tout multi-indice $\gamma$ de $\mathbb{N}^{2 m}$ de longueur $|\gamma|$ et tout $\lambda$ de $\mathbb{C}^{m}$,

$$
\left|D^{\gamma} \tilde{\chi}_{N_{j, 2}-k}(\lambda)\right| \leq A_{7}^{|\gamma|}|\gamma| ! M_{|\gamma|}\left(\frac{A_{5}}{2^{m k}}\right)^{|\gamma|} .
$$

Il existe donc, d'après (1.4) et (3.18)-(3.21), une constante $A_{8}>1$ ne dépendant que de la suite $\left\{M_{p}\right\}_{p \geq 0}$, de l'entier $m$ et du compact $K$ telle que l'on ait, pour tout $(z, \zeta, \lambda)$ de $O(1) \times \mathbb{C}^{m}$,

(3.22) $\left|D_{z}^{\alpha} D_{\zeta}^{\beta} D_{\lambda}^{\gamma} \varphi(z, \zeta, \lambda)\right|$

$$
\leq A_{8}^{|\alpha|+|\beta|+|\gamma|}(|\alpha|+|\beta|+|\gamma|) ! M_{|\alpha|+|\beta|+|\gamma|}\left(\frac{1}{2^{k(\zeta)}}\right)^{|\alpha|+|\beta|+m|\gamma|} .
$$

Ceci, compte tenu de (3.15), établit (3.2).

$$
\text { On a enfin }
$$

$$
\frac{\partial}{\partial \bar{z}} \varphi(z, \zeta, \lambda)=\sum_{k=0}^{\infty} \tilde{\chi}_{C_{2-k}}(\zeta) \sum_{j=0}^{m} \frac{\partial}{\partial \bar{z}} \chi_{D_{j, 2}^{\prime}-(k+3)}(z) \tilde{\chi}_{N_{j, 2-(k-1) 3}}(\lambda) .
$$

Or, $\frac{\partial}{\partial \bar{z}} \widetilde{\chi}_{D_{j, r}^{\prime}} \neq 0$ implique

$$
\frac{1}{16(m+1)} r>d\left(z, D_{j, r}^{\prime}\right)>\frac{1}{2} \cdot \frac{1}{16(m+1)} r
$$

et de même, $\tilde{\chi}_{N_{j, r}}(\lambda) \neq 0$ implique

$$
d\left(\lambda, N_{j, r}\right)<A_{5} r^{m} .
$$

L'inégalité (3.23) implique $d\left(z, \Gamma_{j, r}^{\prime}\right)<\frac{1}{16(m+1)} r$ et donc (3.23) et (3.24) entraînent, d'après (3.11),

$$
\left|P_{m}(z, \lambda)\right| \geq A_{6} r^{m}>0 .
$$

Ceci achève la preuve du lemme d'après (3.15) et (3.18).

4. Propostrion. Soit $K$ un compact non vide de $\mathbb{C}$ et $g$ une fonction de classe $C^{\infty}$ dans $\mathbb{C}$, à support compact dans $V(K)$. On suppose que, pour tout entier $p$ positif ou nul, il existe une constante $C(p) \geq 0$ vérifiant

$$
\left|\frac{\partial}{\partial \zeta} g(\zeta)\right| d(\zeta, K)^{-p} \leq C(p) \quad \text { pour tout } \zeta \text { de } V(K) \backslash K \text {. }
$$

Alors on a, pour tout $z$ de $K$ et tout $\lambda$ de $\mathbb{C}^{m}$,

$$
g(z)=P_{m}(z, \lambda) Q(z, \lambda)+\sum_{k=0}^{m-1} z^{k} R_{k}(\lambda)
$$

avec

$$
Q(z, \lambda)=\frac{1}{\pi^{2}} \int_{\zeta \in V(K) \backslash K} \int_{t \in V(K) \backslash K} \frac{\frac{\partial}{\partial \bar{z}} \varphi(t, \zeta, \lambda) \frac{\partial}{\partial \bar{\zeta}} g(\zeta)}{(\zeta-t)(t-z) P_{m}(t, \lambda)} d \sigma(t) d \sigma(\zeta)
$$

et, pour $k$ entier avec $0 \leq k \leq m-1$,

(4.4) $R_{k}(\lambda)$

$$
=\frac{1}{\pi^{2}} \int_{\zeta \in V(K) \backslash K} \int_{t \in V(K) \backslash K} \frac{\frac{\partial}{\partial \bar{z}} \varphi(t, \zeta, \lambda) \frac{\partial}{\partial \zeta} g(\zeta)}{(\zeta-t) P_{m}(t, \lambda)} S_{k}(t, \lambda) d \sigma(t) d \sigma(\zeta) .
$$

Ici, do désigne la mesure de Lebesgue planaire, $\varphi$ est la fonction introduite dans le lemme 3 et les fonctions $S_{k}, 0 \leq k \leq m-1$, sont des polynômes en $t$ dont les coefficients sont des fonctions affines de $\lambda$ qui vérifient

$$
P_{m}(z, \lambda)-P_{m}(t, \lambda)=(z-t) \sum_{k=0}^{m-1} z^{k} S_{k}(t, \lambda) .
$$

Remarque. Bien évidemment, dans (4.3) e't (4.4), l'intégration en $t$

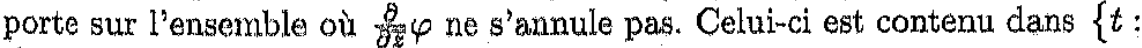
$\left.\frac{1}{64} d(\zeta, K)<d(t, K)<\frac{3}{4} d(\zeta, K)\right\}$.

Preuve de la proposition 4. La formule de Cauchy donne, pour tout $z$ de $\mathbb{C}$

$$
g(z)=-\frac{1}{\pi} \int_{\zeta \in V(K) \backslash K} \frac{\frac{\partial}{\partial \zeta} g(\zeta)}{\zeta-z} d \sigma(\zeta)
$$


D'après (3.1), pour tout $z$ dans $K$, tout $\zeta$ dans $V(K) \backslash K$ et tout $\lambda$ dans $\mathbb{C}^{m}$, on a $\varphi(z, \zeta, \lambda)=1$ et donc

$$
g(z)=-\frac{1}{\pi} \int_{\zeta \in V(K) \backslash K} \frac{\varphi(z, \zeta, \lambda)}{\zeta-z} \cdot \frac{\partial}{\partial \bar{\zeta}} g(\zeta) d \sigma(\zeta) .
$$

Pour $\zeta$ fixé dans $V(K) \backslash K$, la fonction $z \rightarrow \varphi(z, \zeta, \lambda) /(\zeta-z)$ est de classe $C^{\infty}$ et à support compact dans $\left\{z \in \mathbb{C}: d(z, K)<\frac{3}{4} d(\zeta, K)\right\}$. On peut donc appliquer à nouveau la formule de Cauchy et on obtient

$$
\frac{\varphi(z, \zeta, \lambda)}{\zeta-z}=-\frac{1}{\pi} \int_{t \in V(K) \backslash K} \frac{\frac{\partial}{\partial z} \varphi(t, \zeta, \lambda)}{(\zeta-t)(t-z)} d \sigma(t)
$$

On a donc, d'après (4.6) et (4.7),

$$
\text { (4.8) } \quad g(z)=\frac{1}{\pi^{2}} \int_{\zeta \in V(K) \backslash K} \frac{\partial}{\partial \bar{\zeta}} g(\zeta) \int_{t \in V(K) \backslash K} \frac{\frac{\partial}{\partial \bar{z}} \varphi(t, \zeta, \lambda)}{(\zeta-t)(t-z)} d \sigma(t) d \sigma(\zeta) \text {. }
$$

On remarque maintenant que, d'après $(3.3)$, en tout point $(t, \zeta, \lambda)$ où $\frac{\partial}{\partial \bar{z}} \varphi(t, \zeta, \lambda)$ est différent de zéro, $P_{m}(t, \lambda)$ est également différent de zéro. On a alors, dans ce cas,

$$
\frac{1}{t-z}=\frac{P_{m}(z, \lambda)}{(t-z) P_{m}(t, \lambda)}+\sum_{k=0}^{m-1} \frac{z^{k} S_{k}(t, \lambda)}{P_{m}(t, \lambda)}
$$

Les égalités (4.8) et (4.9) donnent la formule de division (4.2).

5. Définitrons et estimations. On note

$$
\mathcal{K}(z, \zeta, \lambda)=\frac{1}{\pi^{2}} \int_{t \in V(K) \backslash K} \frac{\frac{\partial}{\partial \ddot{z}} \varphi(t, \zeta, \lambda)}{(\zeta-t)(t-z) P_{m}(t, \lambda)} d \sigma(t)
$$

et, pour $k$ entier avec $0 \leq k \leq m-1$,

$$
\mathcal{K}_{k}(\zeta, \lambda)=\frac{1}{\pi^{2}} \int_{t \in V(K) \backslash K} \frac{\frac{\partial}{\partial \bar{z}} \varphi(t, \zeta, \lambda)}{(\zeta-t) P_{m}(t, \lambda)} S_{k}(t, \lambda) d \sigma(t)
$$

On rappelle que l'intégration ne porte en fait que sur

$$
\left\{t: \frac{1}{64} d(\zeta, K)<d(t, K)<\frac{3}{4} d(\zeta, K)\right\}
$$

Clairement, d'après $(3.3)$ et $(5.3), \mathcal{K}$ est de classe $C^{\infty}$ dans $O(1 / 64) \times \mathbb{C}^{m}$ et est holomorphe en $z$ pour $(\zeta, \lambda)$ fixé dans $(V(K) \backslash K) \times \mathbb{C}^{m}$. De même, pour tout $k$ avec $0 \leq k \leq m-1, \mathcal{K}_{k}(\zeta, \lambda)$ est de classe $C^{\infty}$ dans $(V(K) \backslash K) \times \mathbb{C}^{m}$.

On déduit des propriétés (3.2) et (3.3) de la fonction $\varphi$ qu'il existe une constante $A_{9}>1$ telle que l'on ait

$$
\left|D_{\lambda}^{L} \frac{\frac{\partial}{\partial \bar{z}} \varphi(t, \zeta, \lambda)}{P_{m}(t, \lambda)}\right| \leq A_{9}^{l} l ! M_{l} d(\zeta, K)^{-(m l+m+1)}
$$

De là, il existe une constante $A_{10}>1$ telle que l'on ait, pour tout entier $j$, tout multi-indice $L$ de $\mathbb{N}^{2 m}$ de longueur $l$ et tout $(z, \zeta, \lambda)$ de $O(1 / 64) \times \mathbb{C}^{m}$

$$
\left|D_{z}^{j} D_{\lambda}^{L} \mathcal{K}(z, \zeta, \lambda)\right| \leq A_{10}^{j+l} l ! j ! M_{l} d(\zeta, K)^{-(m l+j+m+3)} .
$$

et, pour tout multi-indice $L$ de $\mathbb{N}^{2 m}$ de longueur $l$ et pour tout $(\zeta, \lambda)$ de $(V(K) \backslash K) \times \mathbb{C}^{m}$

$$
\left|D_{\lambda}^{L} \mathcal{K}_{k}(\zeta, \lambda)\right| \leq A_{10}^{l} l ! M_{l} d\left(\zeta, K^{\prime}\right)^{-(m l+m+2)}[|\lambda|+1] .
$$

Les constantes $A_{9}$ et $A_{10}$ ne dépendent que de la suite $\left\{M_{p}\right\}_{p \geq 0}$, de l'entier $m$ et du compact $K$.

6. PRopostrion. Soit $K$ un compact non vide de $\mathbb{C}$ et $g$ une fonction de classe $C^{\infty}$ dans $\mathbb{C}$, à support compact dans $V(K)$. On suppose que, pour tout entier $p$ positif ou nul, il existe une constante $C(p) \geq 0$ vérifiant

(6.1) $\quad\left|\frac{\partial}{\partial \bar{\zeta}} g(\zeta)\right| d(\zeta, K)^{-p} \leq C(p) \quad$ pour tout $\zeta$ de $V(K) \backslash K$.

On pose

$$
Q=\left\{Q^{j, L}(z, \lambda): z \in K, \lambda \in \mathbb{C}^{m}, j \in \mathbb{N}, L \in \mathbb{N}^{2 m}\right\}
$$

avec.

$$
Q^{j, L}(z, \lambda)=\frac{1}{\pi^{2}} \int_{\zeta \in V(K) \backslash K} \frac{\partial}{\partial \vec{\zeta}} g(\zeta) D_{z, \lambda}^{j, L} \mathcal{K}(z, \zeta, \lambda) d \sigma(\zeta)
$$

où $\mathcal{K}(z, \zeta, \lambda)$ a été défini en $(5.1)$. On note $R$ la fonction de classe $C^{\infty}$ sur $\mathbb{C} \times \mathbb{C}^{m}$ définie par $(z, \lambda) \rightarrow \sum_{k=0}^{m-1} z^{k} R_{k}(\lambda)$, où $R_{k}(\lambda)$ a été défini en (4.4). Alors les propriétés suivantes sont vérifiées:

(a) $Q$ est un jet de Whitney de classe $C^{\infty}$ sur $K \times \mathbb{C}^{m}, \bar{\partial}$-plat en $z$.

(b) On a, au sens des jets sur $K \times \mathbb{C}^{m}$,

$$
g=P_{m} Q+R
$$

Preuve. On vérifie comme dans la preuve de la proposition 10 de [6] que, d'apres $(5.4)$ et $(6.1), Q$ est un jet de Whitney de classe $C^{\infty}$ sur $K \times \mathbb{C}^{m}$. On peut, à ce point, remanquer que, pour $(j, z)$ fixés dans $\mathbb{N} \times K, Q^{j, L}(z, \lambda)$ est, en fait, la dérivée d'ordre $L$ en $\lambda$ de la fonction de classe $C^{\infty}$ dans $\mathbb{C}^{m}$, $\lambda \rightarrow Q^{j, 0}(z, \lambda)$.

Pour établir (b), pour tout $\varepsilon>0$, on note $\chi_{\varepsilon}$ une fonction continue dans $\mathbb{C}$, à valeurs comprises entre 0 et 1 , égale à 1 sur $\{\zeta \in \mathbb{C}: d(\zeta, K)>\varepsilon\}$ et à $0 \operatorname{sur}\{\zeta \in \mathbb{C}: d(\zeta, K)<\varepsilon / 2\}$. De plus, on pose

(6.4) $g_{\varepsilon}(z)=\frac{1}{\pi^{2}} \int_{\zeta \in V(K) \backslash K} \chi_{\varepsilon}(\zeta) \frac{\partial}{\partial \bar{\zeta}} g(\zeta) \int_{t \in V(K) \backslash K} \frac{\frac{\partial}{\partial \bar{z}} \varphi(t, \zeta, \lambda)}{(\zeta-t)(t-z)} d \sigma(t) d \sigma(\zeta)$, 


$$
\begin{aligned}
Q_{\varepsilon}(z, \lambda) & =\frac{1}{\pi^{2}} \int_{\zeta \in V(K) \backslash K} \chi_{\varepsilon}(\zeta) \frac{\partial}{\partial \bar{\zeta}} g(\zeta) \mathcal{K}(z, \zeta, \lambda) d \sigma(\zeta), \\
Q_{\varepsilon}^{j, L}(z, \lambda) & =\frac{1}{\pi^{2}} \int_{\zeta \in V(K) \backslash K} \chi_{\varepsilon}(\zeta) \frac{\partial}{\partial \bar{\zeta}} g(\zeta) D_{z, \lambda}^{j, L} \mathcal{K}(z, \zeta, \lambda) d \sigma(\zeta)
\end{aligned}
$$

pour tout $j$ et tout $L$,

et, pour $k$ entier avec $0 \leq k \leq m-1$,

$$
R_{k, \varepsilon}(\lambda)=\frac{1}{\pi^{2}} \int_{\zeta \in V(K) \backslash K} \chi_{\varepsilon}(\zeta) \frac{\partial}{\partial \bar{\zeta}} g(\zeta) \mathcal{K}_{k}(\zeta, \lambda) d \sigma(\zeta)
$$

où les fonctions $\mathcal{K}$ et $\mathcal{K}_{k}$ ont été définies en (5.1) et (5.2).

Clairement, puisque dans les intégrales correspondantes n'interviennent que les $\zeta$ vérifiant $d(\zeta, K) \geq \varepsilon / 2$, les fonctions $g_{\varepsilon}, Q_{\varepsilon}$ et $R_{k, \varepsilon}$ sont de classe $C^{\infty}$ sur $K_{\varepsilon / 2}=\{z \in \mathbb{C}: d(z, K)<\varepsilon / 2\}$ et on a, sur $K_{\varepsilon / 2} \times \mathbb{C}^{m}$,

$$
g_{\varepsilon}(z)=P_{m}(z, \lambda) Q_{\varepsilon}(z, \lambda)+\sum_{k=0}^{m_{m}-1} z^{k} R_{k, \varepsilon}(\lambda)
$$

On a clairement, toujours sur $K_{\varepsilon / 2} \times \mathbb{C}^{m}$,

$$
D_{z, \lambda}^{j, L} Q_{\varepsilon}=Q_{\varepsilon}^{j, L} \text {. }
$$

Enfin, on pose

avec

$$
\widetilde{G}^{j}(z)=-\frac{1}{\pi} \int_{\zeta \in V(K) \backslash K} \frac{\partial}{\partial \bar{\zeta}} g(\zeta) D_{z}^{j}\left(\frac{1}{\zeta-z}\right) d \sigma(\zeta) .
$$

On utilise, maintenant, la proposition 10 de [6] pour établir que, lorsque $\varepsilon$ tend vers 0 , le jet de Whitney de classe $C^{\infty}$ défini sur $K \times \mathbb{C}^{m}$ par. $Q_{s}$ tend vers $Q$ dans $C^{\infty}\left(K \times \mathbb{C}^{m}\right)$ et que le jet de Whitney de classe $C^{\infty}$ défini sur $K$ par $g_{\varepsilon}$ tend vers $\widetilde{G}$ dans $C^{\infty}(K)$. Ici encore, on utilise (5.4) et (6.1). De même, on vérifie que $R_{k, \varepsilon}$ tend vers $R_{k}$ dans $C^{\infty}\left(\mathbb{C}^{\text {mn }}\right)$ lorsque $\varepsilon$ tend vers 0 .

On montre, comme dans le paragraphe 5 de [5], en utilisant une approximation de l'unité et (6.1), que, pour tout $z$ dans $K$, on a

$$
\text { (6.11) } \quad \tilde{G}^{j}(z)=D_{z}^{j} g(z) .
$$

On a donc, au sens des jets sur $K$,

$$
\widetilde{G}=g \text {. }
$$

On fait donc tendre $\varepsilon$ vers 0 dans (6.7) et on obtient l'égalité (6.3) au sens des jets sur $K \times \mathbb{C}^{m}$.
7. THÉORÈME DE DIVISION. Soit $K$ un compact non vide de $\mathbb{C}$ et $G$ un jet de Whitney de classe $C^{\infty}$ sur $K, \bar{\partial}$-plat. Alors, il existe $Q$ un jet de Whitney de classe $C^{\infty}$ sur $K \times \mathbb{C}^{m}$, $\bar{\partial}$-plat en $z$ et des fonctions $R_{k}$, $0 \leq k \leq m-1$, de classe $C^{\infty}$ dans $\mathbb{C}^{m}$ tels que l'on ait, au sens des jets de Whitney de classe $C^{\infty}$ sur $K \times \mathbb{C}^{n}$,

$$
G=P_{m} Q+R
$$

Ici, $R$ désigne la fonction de classe $C^{\infty}$ définie sur $\mathbb{C} \times \mathbb{C}^{m}$ par $(z, \lambda) \rightarrow$ $\sum_{k=0}^{m-1} z^{k} R_{k}(\lambda)$

Preuve. Il existe, d'après le théorème de Whitney, une fonction $g$ de classe $C^{\gamma \infty}$ dans $\mathbb{C}$, à support compact dans $V(K)$, telle qu'au sens des jets sur $K$ on ait $g=G$. Puisque $G$ est $\bar{\partial}$-plat sur $K$, la fonction $g$ vérifie (6.1) et on applique la proposition 6 . Le jet $Q$ et les fonctions $R_{k}$ sont définis respectivement par les formules (6.2) et (4.4).

8. Applicatrons. (a) Si $K$ est un compact à bord Lip1, l'ensemble des jets de Whitney de classe $C^{\infty}$ sur $K, \bar{\partial}$-plats s'identifie à $A^{\infty}(K)$, l'ensemble des fonctions holomorphes à l'intérieur de $K$ et $C^{\infty}$ jusqu'au bord. On a donc un théorème de division dans $A^{\infty}(K)$. L'hypothèse " $K$ est un compact à bord Lipl" peut être remplacée par une hypothèse plus faible de type régularité de Whitney.

(b) Si le compact $K^{\prime}$ de $\mathbb{C}$ est un intervalle $I$ de $\mathbb{R}$, l'ensemble des jets de Whitney de classe $C^{\infty}$ sur $K, \bar{\partial}$-plats s'identifie à l'ensemble des fonctions $C^{\infty}$ sur $I$; on retrouve alors un résultat classique de division pour les fonctions de classe $C^{\infty}$ à support compact sur $\mathbb{R}$ (G. Glaeser, S. Lojasiewicz, J. Mather et L. Nirenberg [15], B. Malgrange [11]).

9. Définitions. Soit $C \geq 1$. On note $\left(p ! M_{p} ; C\right)$ la classe des fonctions $f$ indéfiniment dérivables dans $\mathbb{R}^{2}$ vérifiant

$$
\sup _{p} \frac{1}{C^{p} M_{p}}\left(\sup _{x \in \mathbb{R}^{2},|p|=p} \frac{\left|D^{P} f(x)\right|}{p !}\right)=\|f\|_{\left(p ! M_{p} ; C\right)}=\|f\|<\infty .
$$

La classe $\left(p ! M_{p} ; C\right)$ est un espace de Banach.

Soit $K$ un compact à bord Lipl. On note de même $\left(p ! M_{p} ; C\right)_{K}$ l'espace de Banach défini de façon analogue.

On note

$$
\left(p ! M_{p} ; C\right)_{K}^{+}=\left(p ! M_{p} ; C\right)_{K} \cap H\left(K^{\circ}\right)
$$

où $H\left(K^{\circ}\right)$ désigne l'ensermble des fonctions holomorphes dans l'intérieur $K^{\circ}$ de $K$.

Soit $K$ un compact arbitraire et $F$ un jet sur $K$. On pose

$$
L_{0}(F)=\sup _{x \in K}\left|F^{0}(x)\right|
$$


et, pour $p \geq 1$

$$
L_{p}(F)=\max \left(\sup _{|P|=p, x \in K} \frac{\left|F^{P}(x)\right|}{p !}, \sup _{|,|=j \leq p-1,(x, \zeta) \in K \times K} \frac{\left|R_{\zeta}^{J, p-1} F(x)\right|}{j !|\zeta-x|^{p-j}}\right) .
$$

On note $\left\{p ! M_{p} ; C\right\}_{K}$ l'espace de Banach des jets de Whitney $F$ sur $K$ vérifiant

$$
\sup _{p} \frac{L_{p}(F)}{C^{p} M_{p}}=\|F\|_{\left\{p ! M_{p} ; C\right\}_{K}}=\|F\|_{<\infty} .
$$

On note enfin $\left\{p ! M_{p} ; C\right\}_{K}^{+}$le sous-ensemble de $\left\{p ! M_{p} ; C\right\}_{K}$ formé des jets $\bar{\partial}$-plats.

Remarque. Soit $K$ un compact à bord Lipl. A une fonction $f$ de $\left(p ! M_{p} ; C\right)_{K}$, on associe son jet $\mathcal{J f}$ sur $K$; on définit ainsi une injection naturelle $\mathcal{J}$ de $\left(p ! M_{p} ; C\right)_{K}$ dans $\left\{p ! M_{p} ; A_{11} C\right\}_{K}$, où la constante $A_{11} \geq 1$ ne dépend que du compact $K$. On a, de plus,

$$
\|\mathcal{J} f\|_{\left\{p ! M_{p} ; A_{11} C\right\}_{K}} \leq\|f\|_{\left(p ! M_{p} ; C\right)_{K}} .
$$

Dans l'autre direction, à un jet $F$ de $\left\{p ! M_{p} ; C\right\}_{K}$, on associe $F^{0}$; on définit ainsi une injection de $\left\{p ! M_{p} ; C\right\}_{K}$ dans $\left(p ! M_{p} ; C\right)_{K}$ et on a

$$
\left\|F^{0}\right\|_{\left(p ! M_{p} ; C\right)_{K}} \leq\|F\|_{\left\{p ! M_{p} ; C\right\}_{K}} .
$$

On a bien évidemment, pour toute fonction $f,(\mathcal{J} f)^{0}=f$ sur $K$.

On peut remplacer l'hypothèse " $K$ est un compact à bord Lipl" par l'hypothèse " $K$ est Whitney 1-régulier", c'est-à-dire, il existe une constante positive $\gamma$ telle que deux points quelconques $x$ et $y$ de $K$ puissent être joints par un arc rectifiable $\sigma$ contenu dans l'intérieur de $K$, sauf peut être pour un nombre fini de points, et dont la longueur $|\sigma|$ vérifie $|\sigma| \leq \gamma|x-y|$. Cette notion intervient dans des problèmes de prolongement de jets [12], [13].

10. LEMME [9]. Soit $\left\{N_{p}\right\}_{p \geq 0}$ une suite de réels vérifiant (1.1) et (1.2). Soit $C \geq 1$ un réel. Il existe une constante $E$ et un opérateur linéaire continu $T$ de $\left\{p ! N_{p} ; C\right\}_{K}^{+}$dans $C^{\infty}\left(\mathbb{R}^{2}\right)$ tels que l'on ait

$$
T F=F \text { sur } K \text {, }
$$

$$
\begin{gathered}
T F \text { est à support dans } V(K), \\
\left|\frac{\partial}{\partial \zeta} T F(\zeta)\right| d(\zeta, K)^{-p} \leq\|F\|(E C)^{p} N_{p+1},
\end{gathered}
$$

pour tout entier $p$ et tout $\zeta$ de $V(K) \backslash K$.

11. THÉORÈME DE DIVISION. Soit $K$ un compact non vide de $\mathbb{C}$ et soit $\left\{N_{p}\right\}_{p \geq 0}$ une suite de réels vérifiant (1.1) et (1.2). Alors, étant donné $C \geq 1$, il existe des opérateurs linéaires $\mathcal{Q}$ et $\mathcal{R}_{k}, 0 \leq k \leq m-1$, continus

(11.1) $\mathcal{Q}:\left\{p ! N_{p} ; C\right\}_{K}^{+} \rightarrow C^{\infty}\left(K \times \mathbb{C}^{m}\right), \quad \mathcal{R}_{k}:\left\{p ! N_{p} ; C\right\}_{K}^{+} \rightarrow C^{\infty}\left(\mathbb{C}^{m}\right)$, vérifiant les propriétés suivantes: (a) Le jet $\mathcal{Q} F$ est $\bar{\partial}$-plat en $z$.

(b) On a, au sens des jets sur $K \times \mathbb{C}^{m}$,

$$
F=P_{m} \mathcal{Q} F+R
$$

où $R$ désigne la fonction de classe $C^{\infty}$ définie sur $\mathbb{C} \times \mathbb{C}^{m}$ par $(z, \lambda) \rightarrow$ $\sum_{k=0}^{m-1} z^{k i} \mathcal{R}_{k} F(\lambda)$.

Preuve. Soit $\left\{M_{p}\right\}_{p \geq 0}$ une suite de réels vérifiant $(1.1)$ et $(1.3)$ et $F$ appartenant è $\left\{p ! N_{p} ; C\right\}_{K}^{+}$. D'après $(10.2)$ et $(10.3), g=T F$ vérifie les hypothèses de la proposition 4 . On utilise la construction précédemment décrite où la suite $\left\{M_{p}\right\}_{p \geq 0}$ intervient dans la définition de la fonction $\varphi$. On a donc, d'après (10.1), la formule de division (11.2) avec $\mathcal{Q} F$ le jet défini par

$$
\begin{aligned}
(Q F)^{j, L}(z, \lambda)= & \frac{1}{\pi^{2}} \int_{\zeta \in V(K) \backslash K} \frac{\partial}{\partial \bar{\zeta}} T F(\zeta) \\
& \times D_{z, \lambda}^{j, L} \int_{t \in V(K) \backslash K} \frac{\frac{\partial}{\partial \bar{z}} \varphi(t, \zeta, \lambda)}{(\zeta-t)(t-z) P_{m}(t, \lambda)} d \sigma(t) d \sigma(\zeta)
\end{aligned}
$$

et, pour $k$ entier $0 \leq k \leq m-1$,

(11.6) $\quad\left(\mathcal{R}_{k} F\right)(\lambda)$

$$
=\frac{1}{\pi^{2}} \int_{\zeta \in V(K) \backslash K t \in V(K) \backslash K} \int_{\frac{\partial}{\partial \bar{z}} \varphi(t, \zeta, \lambda) \frac{\partial}{\partial \zeta} T F(\zeta)} S_{k}(t, \lambda) d \sigma(t) d \sigma(\zeta) .
$$

12. Estimations. (a) Soit $K$ un compact à bord Lip1. Pour toute fonction $f$ de $\left(p ! N_{p} ; C\right)_{K}^{+},(\mathcal{Q J f})^{0,0}$ est une fonction de $C^{\infty}\left(K \times \mathbb{C}^{m}\right)$ holomorphe en $z$ dans l'intérieur de $K$. On a les estimations suivantes.

Pour tout entier $j$, tout multi-indice $L$ de $\mathbb{N}^{2 m}$ de longueur $l$ et tout $(z, \lambda)$ de $K \times \mathbb{C}^{m}$, on a

$$
\text { (12.1) } \quad\left|D_{z}^{j} D_{\lambda}^{L}(\mathcal{Q} \mathcal{J} f)^{0,0}(z, \lambda)\right| \leq\|f\| A_{12}^{j+l} j ! l ! M_{l} N_{m l+j+m+4}
$$

et, pour tout multimindice $L$ de $\mathbb{N}^{2 m}$ de longueur $l$, tout $\lambda$ de $\mathbb{C}^{m}$ et tout $k$ avec $0 \leq k \leq m-1$, on a

(12.2) $\quad\left|D_{\lambda}^{L}\left(\mathcal{R}_{k} \mathcal{J} f\right)(\lambda)\right| \leq\|f\| A_{12}^{l} l ! M_{l} N_{m l+m+3}[|\lambda|+1]$

Ici, la constante $A_{12}$ dépend de $\left\{M_{p}\right\}_{p \geq 0}$, de $\left\{N_{p}\right\}_{p \geq 0}$, de l'entier $m$, du compact $K$ et de $C$.

Les inégalités (12.1) et (12.2) se déduisent de (10.3), (5.4) et (5.5).

(b) Soit $K$ un compact quelconque. On a des estimations analogues à (12.1) et (12.2) en utilisant la proposition 10 et le corollaire 11 de [6]. Pour tout multi-indice $L$ de $\mathbb{N}^{2 m}$ et tout $\lambda$ de $\mathbb{C}^{m}$,
(12.3)
$(\mathcal{Q} F)^{L}(\lambda)=\left\{(\mathcal{Q} F)^{j, L}(z, \lambda): z \in K, j \in \mathbb{N}\right\}$ 
est un jet de Whitney sur $K$ appartenant à $\left\{p ! N_{m l+p+m+4} ; A_{13} C\right\}_{K}^{+}$et on a (12.4)

$$
\left\|(Q F)^{L}(\lambda)\right\| \leq A_{13}^{l} l ! M_{l}\|F\| .
$$

Ici, les normes sont prises dans les espaces de jets respectifs et la constante $A_{13}$ dépend de $\left\{M_{p}\right\}_{p \geq 0}$, de $\left\{N_{p}\right\}_{p \geq 0}$, de l'entier $m$, du compact $K$ et de $C$.

L'inégalité (12.4) remplace (12.1) et l'inégalité (12.2) n'est pas modifiée.

13. ThÉorème de Division $C^{\infty}$ À paramètres aU SENS Des Jets. Soit $K$ un compact non vide de $\mathbb{C}$ et $B$ une boule euclidienne fermée dans $\mathbb{R}^{s}$. Soit $F$ un jet de Whitney de classe $C^{\infty}$ sur $K \times B$ et $\bar{\partial}$-plat sur $K$. Alors il existe un jet de Whitney $Q$ de classe $C^{\infty}$ sur $K \times \mathbb{C}^{m} \times B, \bar{\partial}$-plat sur $K$ et des fonctions $R_{k}, 0 \leq k \leq m-1$, de classe $C^{\infty}$ sur $\mathbb{C}^{m} \times B$ tels que l'on ait, au sens des jets sur $K \times \mathbb{C}^{m} \times B$,

$$
F=P_{m} Q+R \text {. }
$$

Ici $R$ désigne la fonction de classe $C^{\infty}$ définie sur $\mathbb{C} \times \mathbb{C}^{m} \times B$ par $(z, \lambda, t) \rightarrow$ $\sum_{k=0}^{m-1} z^{k} R_{k}(\lambda, t)$.

Pr e u ve. Le théorème 13 est une conséquence du théorème 11. Sa preuve un peu technique n'est pas donnée ici. Le lecteur familier du langage des jets l'écrira sans peine en adaptant la preuve donnée ci-dessous du théorème 14.

Comme application du théorème 13 , on retrouve le résultat classique suivant [10], [11], [14], [15] dont on donne une preuve à partir du théorème 11.

14. THÉORÈME DE DIVISION. Soit I un intervalle compact de $\mathbb{R}$ et $B$ une boule euclidienne fermée dans $\mathbb{R}^{s}$. Soit $f$ une fonction de classe $C^{\infty}$ sur $I \times B$. Alors il existe une fonction $Q$ de classe $C^{\infty}$ sur $I \times \mathbb{C}^{m} \times B$ et des fonctions $R_{k}, 0 \leq k \leq m-1$, de classe $C^{\infty}$ sur $\mathbb{C}^{m} \times B$ telles que l'on ait

$$
f(x, t)=P_{m}(x, \lambda) Q(x, \lambda, t)+\sum_{k=0}^{m-1} x^{k} R_{k}(\lambda, t)
$$

Preuve. Soit $f$ une fonction de classe $C^{\infty}$ sur $I \times B$. Pour $t$ fixé dans $B$, on note $f_{t}$ la fonction définie sur $I$ par $f_{t}(x)=f(x, t)$.

On se propose de montrer qu'il existe une suite $\left\{N_{p}\right\}_{p \geq 0}$ vérifiant (1.1) et (1.2) telle que la fonction $t \rightarrow f_{t}$ soit une fonction de classe $C^{\infty}$ sur $B$ à valeurs dans l'espace de Banach $\left\{p ! N_{p} ; l\right\}_{I}^{+}$. Pour cela, on établit d'abord que l'on a, pour tout entier $j$, tout multi-indice $T$ de $\mathbb{N}^{s}$ de longueur $\tau$, tout $x$ dans $I$ et tout $t$ dans $B$,

(14.2) $\left|D_{x}^{j}\left(D_{t}^{T} f(x, t)\right)\right| \leq C(\tau) j ! N_{j}$, c'est-à-dire, $\left\|D_{t}^{T} f\right\|_{\left(j ! N_{j} ; \mathfrak{l}\right)_{I}} \leq C(\tau)$.

On note

$$
\tilde{L}_{p}=\sup _{(j, \tau), j+\tau=p}\left(\sup _{T:|T|=r, x \in I, t \in B} \frac{\left|D_{x}^{j} D_{t}^{T} f(x, t)\right|}{j ! \tau !}\right) .
$$

Il existe clairement une suite $\left(\widetilde{M}_{p}\right)_{p \geq 0}$ croissante, vérifiant (1.1) et (1.2) et telle que l'on ait, pour tout $p, \widetilde{L}_{p} \leq \widetilde{M}_{p}$. On a donc, pour tout $(j, \tau) \in \mathbb{N} \times \mathbb{N}$, $\widetilde{M}_{j+\tau} \leq \max \left(\widetilde{M}_{2 j}, \widetilde{M}_{2 \tau}\right) \leq \widetilde{M}_{2 j} \widetilde{M}_{2 \tau}$. De là, si on pose $N_{p}=\widetilde{M}_{2 p}$, on a l'inégalité (14.2) avec $C(\tau)=\tau ! N_{\tau}$.

Puisqu'il existe une constante $A_{14} \geq 1$ et une injection naturelle continue de $\left(j ! N_{j} ; 1\right)_{I}$ dans $\left\{j ! N_{j} ; A_{14}\right\}_{I}^{+}$on obtient le résultat annoncé en remplaçant $N_{j}$ par $A_{14}^{j} N_{j}$.

Avec la suite $\left\{N_{p}\right\}_{p \geq 0}$ ainsi choisie, on applique maintenant à $f_{t}$ le théorème 11. On note

$$
Q(x, \lambda, t)=Q f_{t}(x, \lambda) \quad \text { et } \quad R_{k}(\lambda, t)=\mathcal{R}_{k} f_{t}(\lambda) .
$$

On a alors la formule de division et, puisque les opérateurs $\mathcal{Q}$ et $\mathcal{R}_{k}$ sont linéaires continus, les régularités annoncées.

Toujours comme application du théorème 13, dans le cas particulier où $K$ est un compact à bord Lip1 on a le théorème suivant.

15. ThúorìmE. Soit $K$ un compact de $\mathbb{C}$ à bord Lip1 et $B$ une boule euclidienne fermée dans $\mathbb{R}^{s}$. Soit $F$ une fonction de classe $C^{\infty}$ sur $K \times B$ et holomorphe à l'intérieur de $K$. Alors il existe une fonction $Q$ de classe $C^{\infty}$ sur $K \times \mathbb{C}^{m} \times B$, holomorphe à l'intérieur de $K$ et des fonctions $R_{k}$, $0 \leq k \leq m-1$, de classe $C^{\infty}$ sur $\mathbb{C}^{m} \times B$ tels que l'on ait, sur $K \times \mathbb{C}^{m} \times B$

$$
f(z, t)=P_{m}(z, \lambda) Q(z, \lambda, t)+\sum_{k=0}^{m-1} z^{k} R_{k}(\lambda, t) .
$$

Remarque. Dans le cas particulier où $K$ est le demi-disque de $\mathbb{C}$ $K=\{z \in \mathbb{C}:|z| \leq 1, \operatorname{Im} z \geq 0\}$, on retrouve un résultat de $\mathrm{S}$. Bell et $\mathrm{D}$. Catlin [1], [2].

\section{Classes fortement non quasi-analytiques}

16. Définitions ET NOTATIONS. On suppose que la suite $\left\{M_{p}\right\}_{p \geq 0}$ vérifie, de plus, la condition suivante : il existe une constante $B_{0}>1$ telle que l'on ait

$$
M_{p} \leq B_{0}^{p} M_{j} M_{p-j}, \quad 0 \leq j \leq p
$$

$$
\sum_{k \geq p} \frac{M_{k}}{(k+1) M_{k+1}} \leq B_{0} \frac{M_{p}}{M_{p+1}} \quad \text { pour tout } p \geq 1
$$

Cette dernière hypothèse qui implique évidemment (1.3) est encore appelée hypothèse de forte non quasimanalyticité. Elle intervient de manière essentielle dans le lemme 18. 
On pose, maintenant, pour tout $r>0$,

$$
h_{M}(r)=\inf _{k} M_{k} r^{k}
$$

Clairement, la fonction $h_{M}$ est croissante, strictement positive et inférieure à 1 .

On sait, d'après (16.1) et (16.2), que, quel que soit l'entier $s>1$, il existe une constante $B_{1}<1$ ne dépendant que de $B_{0}$ et de $s$ telle que l'on ait, pour tout $t>0$,

$$
\text { (16.4) } \quad h_{M}\left(B_{1} t\right) \leq h_{M}(t)^{s} \text {. }
$$

17. Remarque. On s'assure aisément que, si $\left\{N_{p}\right\}_{p \geq 0}$ est une suite vérifiant (1.1), (16.1) et (16.2) et si $m$ est un entier strictement positif, alors la suite $\left\{M_{p}\right\}_{p \geq 0}$ définie, pour tout $p$, par

$$
\text { (17.1) } \quad M_{p}=N_{p}^{m}
$$

vérifie aussi (1.1), (16.1) et (16.2). De plus, d'après (16.3) et (16.4), il existe une constante $B_{2}$ dépendant de $m$ et de la suite $\left\{N_{p}\right\}_{p \geq 0}$ telle que l'on ait, pour tout $r>0$,

$$
h_{N}\left(B_{2} r^{1 / m}\right) \leq h_{M}(r) \leq h_{N}\left(r^{1 / m}\right) .
$$

18. LEMME [4], [7]. Soit $\left\{M_{p}\right\}_{p \geq 0}$ une suite vérifiant (1.1), (16.1) et (16.2), $\Omega$ un sous-ensemble non vide de $\mathbb{R}^{s}$, et $\eta$ et a des réels strictement positifs. Il existe une fonction $\chi_{\Omega, \eta}$ de classe $C^{\infty}$ dans $\mathbb{R}^{s} \dot{a}$ valeurs positives ou nulles et vérifiant les propriétés suivantes:

$$
\begin{array}{ll}
\chi_{\Omega, \eta}(x)=1 & \text { si } d(x, \Omega) \leq \frac{1}{2} a, \\
\chi_{\Omega, \eta}(x)=0 & \text { si } d(x, \Omega) \geq a,
\end{array}
$$

Pour tout multi-indice $L$ de $\mathbb{N}^{s}$ de longueur l et tout $x$ de $\mathbb{R}^{s}$, on a

$$
\left|D^{L} \chi_{\Omega, \eta}(x)\right| \leq \eta^{l} l ! M_{l} \frac{1}{h_{M}\left[B_{3} \eta a\right]} .
$$

Ici $B_{3}$ est une constante qui ne dépend que de la dimension s et de la suite $\left\{M_{p}\right\}_{p \geq 0}$.

19. LEMME D'EXTENSION LINÉAIRE [7]. Etant donnée une suite $\left\{N_{p}\right\}_{p \geq 0}$ vérifiant (1.1), (16.1) et (16.2), il existe un opérateur linéaire d'extension continu $T$ de l'espace de Banach $\left\{p ! N_{p} ; C\right\}_{K}$ dans $\left(p ! N_{p} ; E C\right)$. La constante $E$ ne dépend que de la suite $\left\{N_{p}\right\}_{p \geq 0}$; par contre, l'opérateur $T$ dépend aussi de $C$.

20. THÉORÈME DE DIVISION. Soit $K$ un compact non vide et soit $\left\{N_{p}\right\}_{p \geq 0}$ une suite vérifiant (1.1), (16.1) et (16.2). Alors, étant donné $C \geq 1$, il existe des opérateurs linéaires $\mathcal{Q}$ et $\mathcal{R}_{k}, 0 \leq k \leq m-1$, continus

(20.1) $\mathcal{Q}:\left\{p ! N_{p} ; C\right\}_{K}^{+} \rightarrow C^{\infty}\left(K \times \mathbb{C}^{m}\right), \quad \mathcal{R}_{k}:\left\{p ! N_{p} ; C\right\}_{\mathcal{K}}^{+} \rightarrow C^{\infty}\left(\mathbb{C}^{m}\right)$, vérifiant les propriétés suivantes: (a) Le jet $\mathcal{Q} F$ est $\bar{\partial}$-plat en $z$.

(b) On a, au sens des jets sur $K \times \mathbb{C}^{m}$,

$$
F=P_{m} \mathcal{Q F}+R,
$$

où $R$ désigne la fonction de classe $C^{\infty}$ définie sur $\mathbb{C} \times \mathbb{C}^{m}$ par $(z, \lambda) \rightarrow$ $\sum_{k=0}^{m-1} z^{k} \mathcal{R}_{k} F^{\prime}(\lambda)$.

(c) Pour tout multi-indice $L$ de $\mathbb{N}^{22 n}$ de longueurl et tout $\lambda$ de $\mathbb{C}^{m}$, le jet $(\mathcal{Q F})^{L}(\lambda)$ défini à partir de $\mathcal{Q} F$ comme en (12.3) appartient à $\left\{p ! N_{p} ; B_{4}\right\}_{K}^{+}$ et on a

$$
\left\|\mathcal{Q} F^{L}(\lambda)\right\| \leq\|F\| B_{4}^{l} l ! N_{l}^{m}
$$

et, pour tout multi-indice $L$ de $\mathbb{N}^{2 m}$ de longueurl et tout $\lambda$ de $\mathbb{C}^{m}$, on a

$$
\left|D_{\lambda}^{L}\left(\mathcal{R}_{k} F\right)(\lambda)\right| \leq\|F\| B_{4}^{l} l ! N_{l}^{m}[|\lambda|+1] .
$$

Ici, la constante $B_{4}$ dépend de $\left\{N_{p}\right\}_{p \geq 0}$, de l'entier $m$, du compact $K$ et de $C$.

Preuve. On reprend d'abord la construction faite plus haut avec quelques modifications. Soit $\eta>0$. On construit $\varphi_{\eta}$ comme dans le lemme 3 avec $M_{p}=N_{p}^{m}$ et en remplaçant le lemme 2 par le lemme 18.

On contrôle maintenant les dérivées de $\varphi_{\eta}$ sur $O(1) \times \mathbb{C}^{m}$ de la façon suivante. Il existe, d'après (18.3) et (16.4), une constante $B_{5}<1$ dépendant de $K$, de $\left\{N_{p}\right\}_{p \geq 0}$ et de $m$ et une constante $B_{6}>1$ ne dépendant que de $m$ telle que l'on ait :

- pour tout multi-indice $\alpha$ de $\mathbb{N}^{2}$ de longueur $|\alpha|$ et tout $\zeta$ de $\mathbb{C}$,

$$
\left|D^{\alpha} \tilde{\chi}_{C_{2, k}, \eta \eta}(\zeta)\right| \leq\left(B_{6} \eta\right)^{|\alpha|}|\alpha| ! M_{|\alpha|} \frac{1}{h_{M}\left(B_{5} \eta 2^{-k}\right)},
$$

- pour tout multi-indice $\beta$ de $\mathbb{N}^{2}$ de longueur $|\beta|$ et tout $z$ de $\mathbb{C}$,

$$
\text { (20.6) } \quad\left|D^{\beta \beta} \tilde{\chi}_{D_{j, 2-z_{1}}^{\prime}, \eta^{\prime}}(z)\right| \leq\left(B_{6} \eta\right)^{|\beta|}|\beta| ! M_{|\beta|} \frac{1}{h_{M}\left(B_{5} \eta 2^{-k}\right)}
$$

- pour tout multi-indice $\gamma$ de $\mathbb{N}^{2 m}$ de longueur $|\gamma|$ et tout $\lambda$ de $\mathbb{C}^{m}$,

$$
\text { (20.7) } \quad\left|D^{\gamma} \tilde{\chi}_{N_{d, 2}-k, \eta}(\lambda)\right| \leq\left(B_{6} \eta\right)^{|\gamma|}|\gamma| ! M_{|\gamma|} \frac{1}{h_{M}\left(B_{5} \eta 2^{-m k}\right)} \text {. }
$$

Il existe donc, d'après (3.15), (16.4) et (20.5) - (20.7), une constante $B_{7}<1$ ne dépendant que de la suite $\left\{N_{p}\right\}_{p \geq 0}$, de l'entier $m$ et du compact $K$ et une constante $B_{8}>1$ ne dépendant que de $m$ telles que l'on ait, pout tout $(z, \zeta, \lambda)$ de $O(1) \times \mathbb{C}^{m}$

(20.8) $\left|D_{z}^{\alpha} D_{\zeta}^{\beta} D_{\lambda}^{\gamma} \varphi_{\eta}(z, \zeta, \lambda)\right|$

$$
\left.\leq\left(B_{8} \eta\right)^{|\alpha|+|\beta|+|\gamma|}(|\alpha|+|\beta|+|\gamma|)\right) \frac{N_{|\alpha|+|\beta|+|\gamma|}^{m}}{h_{M}\left(B_{7} \eta 2^{-m k(\zeta)}\right)}
$$


et donc, d'après (3.15) et (17.2), quitte à changer $\eta$ en $\eta / B_{8}$,

(20.9) $\left|D_{z}^{\alpha} D_{\zeta}^{\beta} D_{\lambda}^{\gamma} \varphi_{\eta}(z, \zeta, \lambda)\right|$

$$
\leq \eta^{|\alpha|+|\beta|+|\gamma|}(|\alpha|+|\beta|+|\gamma|) ! \frac{N_{|\alpha|+|\beta|+|\gamma|}^{m}}{h_{N}\left(B_{9} \eta^{1 / m} d(\zeta, K)\right)},
$$

où $B_{9}<1$ est une constante ne dépendant que de la suite $\left\{N_{p}\right\}_{p \geq 0}$, de l'entier $m$ et du compact $K$.

Pour tout multi-indice $L$ de $\mathbb{N}^{2 m}$ de longueur $l$ et tout $(z, \zeta, \lambda)$ de $O(1) \times$ $\mathbb{C}^{m}$, on a

$$
\begin{aligned}
& \left|D_{\lambda}^{L} \frac{\frac{\partial}{\partial \bar{z}} \varphi_{\eta}(z, \zeta, \lambda)}{P_{m}(z, \lambda)}\right| \\
& \quad=\sum_{\substack{J, 0 \leq J \leq L \\
|J|=j}} \frac{L !}{J !(L-J) !} D_{\lambda}^{J}\left(\frac{\partial}{\partial \bar{z}} \varphi_{\eta}(z, \zeta, \lambda)\right) D_{\lambda}^{L-J}\left(\frac{1}{P_{m}(z, \lambda)}\right)
\end{aligned}
$$

et donc, d'après (3.3) et (20.9),

$$
\begin{aligned}
& \left|D_{\lambda}^{L} \frac{\frac{\partial}{\partial \bar{z}} \varphi_{\eta}(z, \zeta, \lambda)}{P_{m}(z, \lambda)}\right| \\
& \quad \leq \sum_{\substack{J, 0 \leq J \leq L \\
|\bar{J}|=j}} \frac{L !}{J !(L-J) !} \cdot \frac{\eta^{j+1}(j+1) ! N_{j+1}^{m}}{h_{N}\left(B_{9} \eta^{1 / m} d(\zeta, K)\right)} \cdot \frac{B_{10}^{l-j}(l-j) !}{d(\zeta, K)^{m(l-j+1)}} \\
& \quad \leq B_{11}^{l} \frac{l !}{h_{N}\left(B_{9} \eta^{1 / m} d(\zeta, K)\right)} \sum_{j \leq l} \eta^{j+1} N_{j+1}^{m} \frac{1}{d(\zeta, K)^{m(l-j+1)}}
\end{aligned}
$$

Par définition de $h_{N}$ en (16.3), on a

$$
\left(h_{N}\left(B_{9} \eta^{1 / m} d(\zeta, K)\right)\right)^{m} \leq d(\zeta, K)^{m(l-j+1)} B_{9}^{m(l-j+1)} \eta^{(l-j+1)} N_{l-j+1}^{m}
$$

et donc, d'après (16.4) et (1.1), on obtient

$$
\begin{aligned}
\left|D_{\lambda}^{L} \frac{\frac{\partial}{\partial \bar{z}} \varphi_{\eta}(z, \zeta, \lambda)}{P_{m}(z, \lambda)}\right| \leq & B_{11}^{l} \frac{l !}{h_{N}\left(B_{9} \eta^{1 / m} d(\zeta, K)\right)} \\
& \times \sum_{j \leq l} \eta^{j+1} N_{j+1}^{m} \frac{B_{9}^{m(l-j+1)} \eta^{(l-j+-1)} N_{l-j+1}^{m}}{\left(h_{N}\left(B_{9} \eta^{1 / m} d(\zeta, K)\right)\right)^{m}} \\
\leq & B_{12}^{l} \frac{l ! \eta^{l+2} N_{l+2}^{m}}{h_{N}\left(B_{13} \eta^{1 / m} d(\zeta, K)\right)} .
\end{aligned}
$$

Ici, les constantes $B_{11}>1, B_{12}>1$ et $B_{13}<1$ dépendent de la suite $\left\{N_{p}\right\}_{p \geq 0}$, de l'entier $m$ et du compact $K$.

De là, on a pour $\mathcal{K}$ et $\mathcal{R}_{k}$ des estimations analogues à celles obtenues dans le paragraphe 5 , Il existe donc une constante $B_{14}>1$ ne dépendant que de la suite $\left\{N_{p}\right\}_{p \geq 0}$, de l'entier $m$ et du compact $K$ telle que l'on ait, pour tout entier $j$, tout multi-indice $L$ de $\mathbb{N}^{2 m}$ de longueur $l$ et tout $(z, \zeta, \lambda)$ de $O(1 / 64) \times \mathbb{C}^{m}$,

(20.10) $\left|D_{z}^{j} D_{\lambda}^{L} \mathcal{K}(z, \zeta, \lambda)\right|$

$$
\leq B_{14}^{j+l} l ! j ! \eta^{l+2} N_{l+2}^{m} d(\zeta, K)^{-(j+2)} \frac{1}{h_{N}\left(B_{13} \eta^{1 / m} d(\zeta, K)\right)}
$$

et, pour tout multi-indice $L$ de $\mathbb{N}^{2 m}$ de longueur $l$ et pour tout $(\zeta, \lambda)$ de $(V(K) \backslash K) \times \mathbb{C}^{m}$,

(20.11) $\left|D_{\lambda}^{L} \mathcal{K}_{k}(\zeta, \lambda)\right| \leq B_{14}^{l} l ! N_{l+2}^{m_{2}} \eta^{l+2} d(\zeta, K)^{-1} \frac{[|\lambda|+1]}{h_{N}\left(B_{13} \eta^{1 / m} d(\zeta, K)\right)}$.

Maintenant, par souci de simplicité, on détaille la preuve dans le cas d'un compact $K$ à frontière Lipl.

Soit $f$ une fonction dans $\left(p ! N_{p} ; C\right)_{K}^{-\cdot}$. Alors $T \mathcal{J} f$ définie dans le lemme 19 appartient à $\left(p ! N_{p} ; E C\right)$ et on a

(20.12) $\left|\frac{\partial}{\partial \bar{z}} T \mathcal{J} f(\zeta)\right| \leq\|f\| h_{N}\left(B_{15} d(\zeta, K)\right)$ et donc, d'après (16.4),

$$
\leq\|f\|\left(h_{N}\left(B_{16} d(\zeta, K)\right)\right)^{2},
$$

où $B_{15}$ et $B_{16}$ ne dépendent que de $C$ et de la suite $\left\{N_{p}\right\}_{p>0}$.

De là, on déduit que la fonction $(\mathcal{Q J} f)^{0,0}$ définie par (11.5) vérifie les estimations suivantes:

(20.13) $\left|D_{z}^{j} D_{\lambda}^{L}(\mathcal{Q J} f)^{0,0}(z, \lambda)\right| \leq B_{17}^{j+l} l ! j ! \eta^{l+2} N_{l+2}^{m} d(\zeta, K)^{-(j+2)}$

On choisit

$$
\times \frac{\left(h_{N}\left(B_{16} d(\zeta, K)\right)\right)^{2}\|f\|}{h_{N}\left(B_{13} \eta^{1 / m} d(\zeta, K)\right)} .
$$

On déduit de (20.13), (16.3) et (16.1)

$$
\eta=\left(B_{16} / B_{13}\right)^{m}
$$

(20.14) $\left|D_{z}^{j} D_{\lambda}^{L}(\mathcal{Q J} f)^{0,0}(z, \lambda)\right|$

$$
\begin{aligned}
& \leq\|f\| B_{18}^{j+l} l ! j ! N_{l+2}^{m} d(\zeta, K)^{-(j+2)} h_{N}\left(B_{16} d(\zeta, K)\right) \\
& \leq\|f\| B_{19}^{j+l} l ! j ! N_{l+2}^{m} N_{j+2} \leq\|f\| B_{20}^{j+l} l ! j ! N_{l}^{m} N_{j} .
\end{aligned}
$$

Les constantes $B_{i}, 17 \leq i \leq 20$, ne clépendent que de la suite $\left\{N_{p}\right\}_{p \geq 0}$, de l'entier $m$ et du compact $K$ ot de $C$.

On déduit de même l'estimation correspondante pour $\mathcal{R}_{k} \mathcal{J} f$ défini par (4.4) ou (11.6).

Dans le cas d'un compact $K$ arbitraire, on définit le jet $\mathcal{Q F}$ par la formule (11.5) en utilisant ici encore la fonction $\varphi_{\eta}$ et l'opérateur linéaire continu d'extension $T$ du lemme 19. On obtient alors (c) en utilisant, comme dans le paragraphe 12, la proposition 10 et le corollaire 11 de [6]. 
Comme application du théorème 20 , on a le résultat suivant.

21. THÉORÈME DE DIVISION. Soit $I$ un intervalle compact de $\mathbb{R}$ et $B$ une boule euclidienne fermée dans $\mathbb{R}^{s}$. Soit $f$ une fonction sur $I \times B$, appartenan $\grave{a}\left(p ! N_{p} ; 1\right)_{I \times B}$. Alors il existe une fonction $Q$ de classe $C^{\infty}$ sur $I \times \mathbb{C}^{m} \times B$ et des fonctions $R_{k}, 0 \leq k \leq m-1$, de classe $C^{\infty}$ sur $\mathbb{C}^{m} \times B$ vérifiant les propriétés suivantes:

(a) On a

$$
f(x, t)=P_{m}(x, \lambda) Q(x, \lambda, t)+\sum_{k=0}^{m-1} x^{k} R_{k}(\lambda, t) .
$$

(b) Il existe une constante $B_{21} \geq 1$ telle que l'on ait pour tout entier $j$ tout multi-indice $L$ de $\mathbb{N}^{2 m}$ de longueur $l$, tout multi-indice $T$ de longueur $\tau$ et tout $(x, \lambda, t)$ de $I \times \mathbb{C}^{m} \times B$,

$$
\left|D_{x}^{j} D_{\lambda}^{L} D_{t}^{T} Q(x, \lambda, t)\right| \leq B_{21}^{j+l+\tau} j ! l ! \tau ! N_{j} N_{\tau}\left(N_{l}\right)^{m},
$$

et, pour tout multi-indice $L$ de $\mathbb{N}^{2 m}$ de longueur $l$, tout multi-indice $T$ de longueur $\tau$, tout $(\lambda, t)$ de $\mathbb{C}^{m} \times B$ et tout $k$ avec $0 \leq k \leq m-1$,

$$
\text { (21.3) } \quad\left|D_{\lambda}^{L} D_{t}^{T} R_{k}(\lambda, t)\right| \leq B_{21}^{l+r} l ! \tau ! N_{\tau}\left(N_{l}\right)^{m}[|\lambda|+1]
$$

22. Remarques. (a) Puisque l'on a posé $M_{p}=N_{p}^{m}$, les inégalités (12.4) et (20.3) sont identiques à cela près que la norme du jet $(\mathcal{Q} F)^{L_{L}}$ n'est pas calculée dans le même espace de jets. On constate que l'hypothèse de forte non quasi-analyticité faite sur la suite $\left\{N_{p}\right\}_{p} \geq 0$ permet de rester dans l'espace de jets $\left\{p ! N_{p} ; C\right\}_{K}^{+}$alors que, sans cette hypothèse, on a une perte de régularité de "l'ordre" de $N_{m l}$.

(b) Dans le cas où on a $N_{p}=p !^{\alpha}, \alpha>0$, c'est-à-dire, le cas des classes de Gevrey d'ordre $1+\alpha$, le théorème 21 permet de retrouver un résultat de M. D. Bronshteĭn [3]. Comme l'explique M. D. Bronshteln, les estimations obtenues sont optimales.

Toujours comme application du théorème 20, on a le théorème suivant.

23. THÉorème. Soit $K$ un compact de $\mathbb{C}$ à bord Lip1 et $B$ une boule euclidienne fermée dans $\mathbb{R}^{s}$. Soit $F$ une fonction appartenant $\dot{a}\left(p ! N_{p} ; l\right)_{K \times B}$ et holomorphe à l'intérieur de $K$. Alors il existe une fonction $Q$ de classe $C^{\infty}$ sur $K \times \mathbb{C}^{m} \times B$, holomorphe à l'intérieur de $K$ et des fonctions $R_{k}$, $0 \leq k \leq m-1$, de classe $C^{\infty}$ sur $\mathbb{C}^{m} \times B$ vérifiant les propriétés suivantes:

(a) On a, sur $K \times \mathbb{C}^{m} \times B$,

$$
f(z, t)=P_{m}(z, \lambda) Q(z, \lambda, t)+\sum_{k=0}^{m-1} z^{k} R_{k}(\lambda, t)
$$

(b) Il existe une constante $B_{22} \geq 1$ telle que l'on ait, pour tout entier $j$, tout multi-indice $L$ de $\mathbb{N}^{2 m}$ de longueur $l$, tout multi-indice $T$ de longueur $\tau$ et tout $(z, \lambda, t)$ de $K \times \mathbb{C}^{m} \times B$,

$$
\left|D_{z}^{j} D_{\lambda}^{L} D_{t}^{T} Q(z, \lambda, t)\right| \leq B_{22}^{j+l+\tau} j ! l ! \tau ! N_{j} N_{\tau}\left(N_{l}\right)^{m},
$$

et, pour tout multi-indice $L$ de $\mathbb{N}^{2 m}$ de longueur $l$, tout multi-indice $T$ de longueur $\tau$, tout $(\lambda, t)$ de $\mathbb{C}^{m} \times B$ et tout $k$ avec $0 \leq k \leq m-1$,

$$
\left|D_{\lambda}^{L} D_{t}^{T} R_{k}(\lambda, t)\right| \leq B_{22}^{l+\tau} l ! \tau ! N_{r}\left(N_{l}\right)^{m}[|\lambda|+1]
$$

Applications. On se propose de donner ici quelques résultats du type "Théorème de Newton".

Soit $\left\{N_{p}\right\}_{p \geq 0}$ une suite de réels positifs. On note $\left(p ! N_{p}\right)=\bigcup_{C}\left(p ! N_{p} ; C\right)$ et on définit de même pour un compact $K$ de $\mathbb{C},\left\{p ! N_{p}\right\}_{K}$ et $\left\{p ! N_{p}\right\}_{K}^{+}$.

24. Théorème. Soit $\Pi$ un polynôme de degré $m$ dans $\mathbb{C}$. Soit $K$ un compact de $\mathbb{C}$. On note $K^{\prime}=\Pi^{-1}(K)$. Soit une suite $\left\{N_{p}\right\}_{p \geq 0}$ vérifiant $(1.1),(16.1)$ et $(16.2)$ et $F$ un jet de Whitney sur $K^{\prime}$ appartenant $\dot{a}\left\{p ! M_{p}\right\}_{K^{\prime}}^{+}$ telle que

(24.1) pour tout $\lambda$ de $K, F$ soit constant sur $\Pi^{-1}(\lambda)$.

Alors il existe une fonction $g$ appartenant $\grave{a}\left(p ! N_{p}^{m}\right)$ telle que $\bar{\partial} g$ s'annule $\grave{a}$ l'ordre infini sur $K$ et que l'on ait, au sens des jets sur $K$,

Preuve. On note

$$
F=g \circ \Pi \text {. }
$$

$$
\Pi(z)=z^{m}+a_{m} z^{m-1}+\ldots+a_{1}
$$

et, pour tout $\lambda$ dans $\mathbb{C}$,

$$
P_{m}(z, \lambda)=z^{m}+a_{m} z^{m-1}+\ldots+a_{1}-\lambda .
$$

On applique alors le théorème de division 20 avec pour diviseur le polynôme $P_{m}$ dans lequel le seul paramètre est $\lambda$. Il existe un jet $Q$ sur $K^{\prime} \times \mathbb{C}$ et une fonction $R$ de classe $C^{\infty}$ définie sur $\mathbb{C} \times \mathbb{C}$ par

$$
R(z, \lambda)=\sum_{k=0}^{m-1} z^{k_{i}} r_{k}(\lambda)
$$

tels que l'on ait, au sens des jets sur $K^{\prime}$,

$$
F=P_{m} Q+R
$$

De plus, pour tout multi-indice $L$ de $\mathbb{N}^{2}$ de longueur $l$, tout $\lambda$ de $\mathbb{C}$ et tout $k$ avec $0 \leq k \leq m-1$, on a

$$
\text { (24.4) } \quad\left|D_{\lambda}^{L} r_{k}(\lambda)\right| \leq B_{23}^{l} l ! N_{l}^{m}[|\lambda|+1]
$$

Soit $z$ un point de $K^{\prime}$. On pose $\lambda=\Pi(z)$ dans (24.3). On note $z_{i}, 1 \leq$ $i \leq m$, les racines de l'équation $\Pi(z)=\lambda$, comptées avec leur multiplicité. 
On a donc, pour tout $i$ avec $1 \leq i \leq m$, au sens des jets sur $K^{\prime}$,

$$
F\left(z_{i}\right)=\sum_{k=0}^{m-1} z_{i}^{k} r_{k}(\lambda)
$$

et donc,

$$
m F(z)=\sum_{k=0}^{m-1} r_{k}(I I(z)) \sum_{i=1}^{m} z_{i}^{k} .
$$

On sait bien que, pour chaque $k, \sum_{i=1}^{m} z_{i}^{k}$ peut s'écrire comme un polynôme des fonctions symétriques élémentaires des racines du polynôme $\Pi(z)-\lambda$, donc des $a_{i}, 1 \leq i \leq m$, et de $\lambda$. De là, il existe des polynômes dépendant de $k$ et notés $\mathcal{P}_{k}$ tels que l'on ait, au sens des jets sur $K^{\prime}$,

$$
m F(z)=\sum_{k=0}^{m-1} r_{k}(\Pi(z)) \mathcal{P}_{k}(\Pi(z)) .
$$

D'après (24.6), si on pose

$$
g(u)=\frac{1}{m} \sum_{k=0}^{m-1} r_{k}(u) \mathcal{P}_{k}(u)
$$

on a bien $F=g \circ \Pi$ au sens des jets sur $K^{\prime}$.

La fonction $g$ est définie et de classe $\mathbb{C}^{\infty}$ dans $\mathbb{C} \times \mathbb{C}$. D'après (24.4), elle appartient à $\left(p ! N_{p}^{m}\right)$, quitte à la multiplier par une fonction à support compact dans cette classe. Puisque, par hypothèse, le jet $\bar{\partial} F$ est nul sur $K^{\prime}$, la fonction $\partial(g \circ \Pi) / \partial \bar{z}$ est nulle à l'ordre infini sur $K^{\prime}$. Puisque

$$
\frac{\partial g}{\partial \bar{u}} \overline{\left(\frac{\partial I}{\partial z}\right)}(z)
$$

a toutes ses dérivées nulles sur $K^{\prime}$ et que $\Pi$ a une dérivée d'ordre $m$ toujours différente de 0 , nécessairement $\partial g / \partial \bar{u}$ est identiquement nulle sur $K$.

25. Corollaire. Soit $f$ une fonction paire sur $[-1,1]$ appartenant d la classe de Gevrey $\left(p !^{1+\alpha}\right)_{[-1,1]}, \alpha>0$. Alors il existe $g$, unique, appartenant $\dot{a}\left(p !^{1+2 \alpha}\right)_{[0,1]}$ telle que l'on ait

$$
f(x)=g\left(x^{2}\right) \quad \text { pour tout } x \text { de }[-1,1] .
$$

26. Re marque. La régularité de $g$ dans le corollaire 25 est optimale comme en témoigne l'exemple suivant.

Soit $f$ la fonction définie sur $[-1,1]$ par $f(x)=\exp \left(-1 /|x|^{1 / \alpha}\right)$, prolongée par 0 à l'origine. On sait que $f$ appartient à $\left(p !^{1+\alpha}\right)_{[-1,1]}$. On a, bien évidemment, $f=g \circ \Pi$ sur $[-1,1]$ avec $g$ définie sur $\mathbb{R}$ par $g(x)=$ $\exp \left(-1 /|x|^{1 / 2 \alpha}\right)$, également prolongée par 0 à l'origine. Clairement $g$ appartient à la classe de Gevrey $\left(p !^{1+2 \alpha}\right)$ et n'appartient à aucune classe de Gevrey plus petite.
27. THÉORÈME. Soit une suite $\left\{N_{p}\right\}_{p \geq 0}$ vérifiant (1.1), (16.1) et (16.2). Soit II un polynôme à coefficients réels de degré $m$ et soit $C$ une constante, $C>1$. Il existe alors une constante $C^{\prime}>1$ et des opérateurs linéaires continus $R_{0}, \ldots, R_{m-1}$ de $\left(p ! N_{p} ; C\right)_{[0,1]}$ dans $\left(p ! N_{p}^{m} ; C^{\prime}\right)_{\mathbb{R}}$ tels que, pour toute fonction $f$ de $\left(p ! N_{p} ; C\right)_{[0,1]}$ et tout $x$ de $[0,1]$, on ait

$$
f(x)=\sum_{k=0}^{m-1} x^{k} R_{k} f(\Pi(x))
$$

Preuve. On note $P_{m}(z, \lambda)$ le polynôme défini par $P_{m}(z, \lambda)=\Pi(z)-\lambda$. Le théorème de division 20 établit l'existence d'opérateurs linéaires continus $\mathcal{Q} \operatorname{de}\left(p ! N_{p} ; C\right)_{[0,1]}$ dans $C^{\infty}(K \times \mathbb{C})$ et $\mathcal{R}_{k}, 0 \leq k \leq m-1$, de $\left(p ! N_{p} ; C\right)_{[0,1]}$ dans $C^{\infty}(\mathbb{C})$ tels que, pour toute fonction $f$ de $\left(p ! N_{p} ; C\right)_{[0,1]}$, tout $x$ de $[0,1]$ et tout $\lambda$ de $\mathbb{C}$, on ait

$$
f(x)=P_{m}(x, \lambda) \mathcal{Q} f(x, \lambda)+\sum_{k=0}^{m-1} x^{k} \mathcal{R}_{k} f(\lambda) .
$$

De plus, $\mathcal{Q}$ et $\mathcal{R}_{k}, 0 \leq k \leq m-1$, vérifient les estimations (20.3) et (20.4). $\mathrm{Si}$, dans l'égalité (27.1), on choisit $\lambda=I(x)$ on obtient, pour tout $x$ de $[0,1]$

$$
f(x)=\sum_{k=0}^{m-1} x^{k} \mathcal{R}_{k} f(\Pi(x))
$$

On remarque que, pour tout $x$ de $[0,1], I(x)$ appartient à un intervalle borné de $\mathbb{R}$; soit $[a, b]$ cet intervalle. Il existe alors une fonction $\chi$ de $\left(p ! N_{p}^{m} ; C^{\prime}\right)_{\mathbb{R}}$ à support compact et vérifiant $\chi(\lambda)=1$ pour tout $\lambda$ de $[a, b]$.

On note $R_{k}, 0 \leq k \leq m-1$, les opérateurs de $\left(p ! N_{p} ; C\right)_{[0,1]}$ dans $\left(p ! N_{p}^{m} ; C^{\prime}\right)_{\mathbb{R}}$ définis par

$$
R_{k} f(\lambda)=\chi(\lambda) \mathcal{R}_{k} f(\lambda) .
$$

Les opérateurs $R_{k}, 0 \leq k \leq m-1$, vérifient les conclusions du théorème.

28. Remarques. (a) Dans le théorème 27, la décomposition n'est pas unique. En effet, pour $N_{p}=p !^{\alpha}, 0<\alpha, \Pi(x)=x^{3}$ et $f(x)=$ $\exp \left(-1 /|x|^{1 / \alpha}\right)$, on peut ecrire $f(x)=g\left(x^{3}\right)$ ou $f(x)=x h\left(x^{3}\right)$ avec $g$ et $h$ dans $\left(p !^{1-1+3 \alpha} ; C^{\prime}\right)_{\mathbb{K}}$ pour $C^{\prime}$ bien choisi alors que $f$ appartient à $\left(p !^{1+\alpha} ; C\right)_{[0,1]}$ pour $C$ bien choisi.

(b) Les régularités obtenues dans le théorème 27 et donc les estimations (20.3) et (20.4) sont optimales. En effet, on considère $I I(x)=x^{m}$. Il existe, d'après le lemme 19 , une constante $E \geq 1$ et une fonction $f$ appartenant à $\left(p ! N_{p} ; E\right)_{[0,1]}$ telle que l'on ait $D^{p} f(0)=p ! N_{p}$ pour tout p. S'il existe alors des fonctions $g_{k}$ de classe $C^{\infty}$ sur $[0,1]$ telles que l'on ait $f(x)=\sum_{k=0}^{m-1} x^{k} g_{k}\left(x^{m}\right)$ sur $[0,1]$, on doit avoir, après identification 
des développements de Taylor en $0, \frac{1}{p !} D^{p} g_{k}(0)=N_{p m+k}$. On déduit alors, d'après les hypothèses faites sur la suite $\left(N_{p}\right)_{p \geq 0}$, que les fonctions $g_{k}$, $0 \leq k \leq m-1$, n'appartiennent à $\left(p ! N_{p}^{l}\right)_{[0,1]}$ pour aucun $l$ réel, $l<m$.

\section{Bibliographie}

[1] S. Bell and D. Catlin, Proper holomorphic mappings extend smoothly to the boundary, Bull. Amer. Math. Soc. 7 (1982), 269-272.

[2] - , - Boundary regularity of proper holomorphic mappings, Duke Math. J. 49 (1982), 385-396.

[3] M. D. Bronshte In, Division with remainder in spaces of smoath functions, Trans. Moscow Math. Soc. 52 (1990), 109-138.

[4] J. Bruna, An extension theorem of Whitney type for non-quasianalytic classes of functions, J. London Math. Soc. (2) 22 (1980), 495-505.

[5] J. Chaumat et A. M. Chollet, Noyaux pour résoudre l'équation $\bar{\partial}$ dans des classes ultradifférentiables sur des compacts irréguliers de $\mathbb{C}^{m}$, dans : Several Complex Variables, Proc. Mittag-Leffler Inst. 1987-88, Math. Notes 38, Princeton University Press, 1993, 205-226.

[6] -, -, Représentations intégrales de jets de Whitney, dans: The Madison Symposium on Complex Analysis, Contemp. Math. 137, Amer. Math. Soc., 1992, 133-153.

[7] - -, Théorème de Whitney dans des classes ultradifférentiables, Publ. I.R.M.A. Lille, 1992.

[8] K. Diederich and J. E. Fornæss, Boundary regularity of proper holomorphic mappings, Invent. Math. 67 (1982), 363-384.

[9] E. M. Dynkin, Pseudoanalytic extension of smooth functions. The uniform scale, Amer. Math. Soc. Transl. 115 (1980), 33-58.

[10] L. Hörmander, The Analysis of Linear Partial Differential Operators I, 2ème éd. Springer, 1989.

[11] B. Malgrange, Ideals of Differentiable Functions, Oxford University Press, 1966.

[12] W. Pleśniak, Extension and polynomial approximation of ultradifferentiable functions in $\mathbb{R}^{n}$, Bull. Soc. Roy. Sci. Liège 63 (1994), 393-402,

[13] V. Thilliez, Prolongement dans des classes ultradifférentiables et propriétés de régularité des compacts de $\mathbb{R}^{n}$, Ann. Polon. Math., à paraitre.

[14] J. C. Tougeron, Idéaux de fonctions différentiables, Springer, 1972.

[15]. Proceedings of Liverpool Singularities Symposium I, Lecture Notes in Math. 192, Springer, 1971.

UNIVERSITÉ PARIS-SUD

MATHEMATIQUES, BÂT. 425

91405 ORSAY CEDEX, FRANCE
UNTVERSITTS DE LILLE U.F.R. DQ MATHETMTIQUE 59655 VILLENEUVE D'ASCQ CEDEX, FRANCE

\section{An example of a non-topologizable algebra}

by

R. FRANKIEWICZ and G. PLEBANEK (Wrockaw)

Abstract. We present an example of an algebra that is generated by $\omega_{1}$ elements, and cannot be made a topological algebra. This answers a problem posed by W. Żelazko.

A real or complex algebra $\mathbf{A}$ is said to be topologizable if there exists a topology $\tau$ on $\mathbf{A}$ such that $(\mathbf{A}, \tau)$ is a Hausdorff topological vector space, and multiplication in $\mathbf{A}$ is jointly continuous (see [3]). While one can always find a vector space topology in which multiplication is separately continuous, there are algebras that are not topologizable.

Zelazko [4] showed that $\mathcal{L}(X)$, the algebra of all endomorphisms of a linear space $X$, is not topologizable as a locally convex algebra whenever $X$ is of infinite dimension. Müller [2] gave an example of a commutative algebra that is not topologizable. He also noted that $\mathcal{L}(X)$ is not topologizable at all for infinite-dimensional $X$

On the other hand, Żelazko [5] proved the following positive result on topologization of algebras ( $\tau_{\text {max }}^{\mathrm{LC}}$ denotes the maximal topology, i.e. the topology generated by all seminorms).

THEOREM 1. Let $\mathbf{F}$ be a real or complex free algebra in variables $\left(t_{i}\right.$ : $i \in I)$. Then $\left(\mathbf{F}, \tau_{\text {max }}^{\mathrm{LC}}\right)$ is a (complete) locally convex topological algebra if and only if the set of variables is at most countable. Consequently, every countably generated algebra car be topologized as a locally convex complete topological algebra.

Zelazko [5] noted that, since the above-mentioned examples of nontopologizable algebras are $2^{\omega}$ menerated, the result of Theorem 1 is best possible if the continuum hypothesis holds.

To show that the second statement of Theorem 1 is indeed optimal, at least concerning the number of generators, we present below an example of an $\omega_{1}$-generated algebra that is not topologizable. For this we modify

1991 Mathematics Subject Classification: Primary 46H05.

Research partially supported by KBN grant 2 P 30104307. 